Bull. Korean Math. Soc. 52 (2015), No. 1, pp. 105-123

http://dx.doi.org/10.4134/BKMS.2015.52.1.105

\title{
ON HARMONIC CONVOLUTIONS INVOLVING A VERTICAL STRIP MAPPING
}

\author{
Raj Kumar, Sushma Gupta, Sukhjit Singh, and Michael Dorff
}

\begin{abstract}
Let $f_{\beta}=h_{\beta}+\bar{g}_{\beta}$ and $F_{a}=H_{a}+\bar{G}_{a}$ be harmonic mappings obtained by shearing of analytic mappings

$$
h_{\beta}+g_{\beta}=1 /(2 i \sin \beta) \log \left(\left(1+z e^{i \beta}\right) /\left(1+z e^{-i \beta}\right)\right), 0<\beta<\pi
$$

and $H_{a}+G_{a}=z /(1-z)$, respectively. Kumar et al. [7] conjectured that if $\omega(z)=e^{i \theta} z^{n}(\theta \in \mathbb{R}, n \in \mathbb{N})$ and $\omega_{a}(z)=(a-z) /(1-a z), a \in(-1,1)$ are dilatations of $f_{\beta}$ and $F_{a}$, respectively, then $F_{a} \widetilde{*} f_{\beta} \in S_{H}^{0}$ and is convex in the direction of the real axis, provided $a \in[(n-2) /(n+2), 1)$. They claimed to have verified the result for $n=1,2,3$ and 4 only. In the present paper, we settle the above conjecture, in the affirmative, for $\beta=\pi / 2$ and for all $n \in \mathbb{N}$.
\end{abstract}

\section{Introduction}

Let $\mathcal{H}$ be the class of all complex valued harmonic functions $f$ defined in the unit disk $E=\{z:|z|<1\}$ and normalized by the conditions $f(0)=0$ and $f_{z}(0)=1$. Such harmonic mappings can be decomposed as $f=h+\bar{g}$, where $h$ is known as the analytic and $g$ the co-analytic part of $f$. A harmonic mapping $f=h+\bar{g}$ defined in $E$, is locally univalent and sense-preserving if and only if $h^{\prime} \neq 0$ in $E$ and the dilatation function $\omega$, defined by $\omega=g^{\prime} / h^{\prime}$, satisfies $|\omega(z)|<1$ for all $z \in E$. We denote by $S_{H}$ the class of all univalent and sense-preserving harmonic mappings in $\mathcal{H}$. Function $f=h+\bar{g}$ in the class $S_{H}$ has the representation

$$
f(z)=z+\sum_{n=2}^{\infty} a_{n} z^{n}+\sum_{n=1}^{\infty} \bar{b}_{n} \bar{z}^{n} \text { for all } z \text { in } E .
$$

The class of functions of the type (1), with $b_{1}=0$, is a subset of $S_{H}$ and will be denoted by $S_{H}^{0}$ here. Further, let $K_{H}$ (respectively, $K_{H}^{0}$ ) be the subclass of $S_{H}$ (respectively, $S_{H}^{0}$ ) consisting of functions which map the unit disk $E$ onto convex domains. A domain $\mathbb{D}$ is said to be convex in the direction $\phi, 0 \leq \phi<\pi$,

Received August 28, 2013; Revised August 30, 2014.

2010 Mathematics Subject Classification. 30C45.

Key words and phrases. univalent harmonic mapping, vertical strip mapping, harmonic convolution. 
if every line parallel to the line through 0 and $e^{i \phi}$ has either connected or empty intersection with $\mathbb{D}$. In particular, a domain convex in the direction of the real axis is denoted by CHD. Clunie and Sheil-Small [1] introduced a method, known as 'shear construction or shearing', for constructing a univalent harmonic mapping associated with a pair of analytic mappings . They proved:

Theorem A. A locally univalent harmonic mapping $f=h+\bar{g}$ in $E$ is a univalent mapping of $E$ onto a domain convex in a direction $\alpha$ if and only if $h-e^{2 i \alpha} g$ is a conformal univalent mapping of $E$ onto a domain convex in the direction of $\alpha$.

Although, harmonic mappings are close relatives of conformal mappings, but their behavior is different, in many ways, than that of conformal mappings. For example, the boundary correspondence which holds in the conformal case fails in the harmonic case. Therefore several researchers investigated subclasses of harmonic mappings that map $E$ onto some specific domains. In particular, Hengartner and Sobber [5] investigated the subclass, $S_{H}(E, \Omega)$, of harmonic mappings in $S_{H}$, which map $E$ onto the horizontal strip domain $\Omega=\{w:|\operatorname{Im}(\mathrm{w})|<\pi / 4\}$. Using a rotation and a composition on the mappings of the class $S_{H}(E, \Omega)$, Doff [2] defined, analogously, the family, $S_{H}\left(E, \Omega_{\beta}\right)$, of harmonic mappings in $S_{H}$, which map $E$ onto asymmetric vertical strip domains

$$
\Omega_{\beta}=\left\{w: \frac{\beta-\pi}{2 \sin \beta}<\operatorname{Re}(\mathrm{w})<\frac{\beta}{2 \sin \beta}\right\},
$$

$\pi / 2 \leq \beta<\pi$. Each $f_{\beta}=h_{\beta}+\bar{g}_{\beta} \in S_{H}\left(E, \Omega_{\beta}\right)$ has the form

$$
h_{\beta}+g_{\beta}=\frac{1}{2 i \sin \beta} \log \left(\frac{1+z e^{i \beta}}{1+z e^{-i \beta}}\right) \text {. }
$$

Convolution (or Hadamard product) of two analytic functions $f(z)=z+$ $\sum_{n=2}^{\infty} a_{n} z^{n}$ and $F(z)=z+\sum_{n=2}^{\infty} A_{n} z^{n}$, is denoted by $f * F$, and is defined as

$$
(f * F)(z)=z+\sum_{n=2}^{\infty} a_{n} A_{n} z^{n} .
$$

In case of harmonic mappings, $f(z)=h+\bar{g}=z+\sum_{n=2}^{\infty} a_{n} z^{n}+\sum_{n=1}^{\infty} \bar{b}_{n} \bar{z}^{n}$ and $F(z)=H+\bar{G}=z+\sum_{n=2}^{\infty} A_{n} z^{n}+\sum_{n=1}^{\infty} \bar{B}_{n} \bar{z}^{n}$, we define their convolution as,

$$
(f \widetilde{*} F)(z)=(h * H)(z)+\overline{(g * G)}(z)=z+\sum_{n=2}^{\infty} a_{n} A_{n} z^{n}+\sum_{n=1}^{\infty} \bar{b}_{n} \bar{B}_{n} \bar{z}^{n} .
$$

In general, the behavior of the harmonic convolution is not as nice as that of the analytic convolution. For example, unlike the case of analytic mappings, convolution of two convex harmonic mappings is not necessarily convex harmonic. But still, convolutions of harmonic mappings exhibit some interesting properties. For example, Dorff [3] and Dorff et al. [4] proved that, under some suitable restrictions on the dilatations, harmonic convolution of two harmonic 
right half-plane mappings is CHD. Li and Ponnusamy [8] extended the results of Dorff et al. [4] by considering one of the mappings as slanted right half-plane mapping. Dorff, in the same paper [3], also investigated harmonic convolutions of a right half-plane mapping with mappings $f_{\beta} \in S_{H}\left(E, \Omega_{\beta}\right), \pi / 2 \leq \beta<\pi$, and established the following result.

Theorem B. Let $f=h+\bar{g} \in K_{H}^{0}$ with $h+g=z /(1-z)$. Then $f \widetilde{*} f_{\beta} \in S_{H}^{0}$ and is $C H D$, provided $f \widetilde{*} f_{\beta}$ is locally univalent and sense-preserving in $E$, where $f_{\beta} \in S_{H}\left(E, \Omega_{\beta}\right), \pi / 2 \leq \beta<\pi$.

Later, Dorff et al. [4] proved that the condition, that $f \widetilde{*} f_{\beta}$ is locally univalent and sense-preserving, can be dropped in some special cases. They proved:

Theorem C. Let $f_{\beta}$ be as in Theorem $B$ with dilatation $g_{\beta}^{\prime}(z) / h_{\beta}^{\prime}(z)=e^{i \theta} z^{n}$, $\theta \in \mathbb{R}$. If $F_{0}=H_{0}+\bar{G}_{0}$, where $H_{0}+G_{0}=z /(1-z)$ with dilatation $G_{0}^{\prime}(z) / H_{0}^{\prime}(z)$ $=-z$, then $F_{0} \widetilde{*} f_{\beta} \in S_{H}^{0}$ and is $C H D$ for $n=1,2$.

For some more relevant and interesting results we refer to some recent papers of Li and Ponnusamy [9, 10] and that of Li et al. [11].

Kumar et al. [7] investigated the harmonic convolutions of mapping $f_{\beta}$ with mappings $F_{a}=H_{a}+\bar{G}_{a}$, where

(3) $H_{a}+G_{a}=\frac{z}{1-z} \quad$ with dilatations $\omega_{a}(z)=\frac{a-z}{1-a z}, a \in(-1,1)$

and essentially proved the following result, which generalizes Theorem C:

Theorem D. Let

$$
f_{\beta}=h_{\beta}+\bar{g}_{\beta}
$$

where $h_{\beta}+g_{\beta}=1 /(2 i \sin \beta)\left(\log \left(\left(1+z e^{i \beta}\right) /\left(1+z e^{-i \beta}\right)\right), 0<\beta<\pi\right.$, with $g_{\beta}^{\prime}(z) / h_{\beta}^{\prime}(z)=e^{i \theta} z^{n}, \theta \in \mathbb{R}$. Then $F_{a} \widetilde{*} f_{\beta} \in S_{H}^{0}$ and are CHD, provided $a$ is restricted in the interval $[(n-2) /(n+2), 1)$ whenever $n=1,2,3$ and 4 .

Note that for $a=0$, the mapping $F_{a}$ reduces to the mapping $F_{0}$ of Theorem C. At the end of the above paper [7] authors conjectured the following general result.

Conjecture. Let $f_{\beta}=h_{\beta}+\bar{g}_{\beta}$ be as in Theorem D. Then $F_{a} \widetilde{*} f_{\beta} \in S_{H}^{0}$ and is CHD for all $n \in \mathbb{N}$, provided $a \in[(n-2) /(n+2), 1)$.

In the present paper, we settle this conjecture, in the affirmative, for $\beta=$ $\pi / 2$.

\section{Main results}

The following results will be required in proving our main theorem.

Lemma 2.1 (Cohn's Rule [12, p. 375]). Given a polynomial $t(z)=a_{0}+a_{1} z+$ $a_{2} z^{2}+\cdots+a_{n} z^{n}$ of degree $n$, let

$$
t^{*}(z)=z^{n} \overline{t\left(\frac{1}{\bar{z}}\right)}=\bar{a}_{n}+\bar{a}_{n-1} z+\bar{a}_{n-2} z^{2}+\cdots+\bar{a}_{0} z^{n} .
$$


Denote by $r$ and $s$, the number of zeros of $t$ inside and on the unit circle $|z|=1$, respectively. If $\left|a_{0}\right|<\left|a_{n}\right|$, then

$$
t_{1}(z)=\frac{\bar{a}_{n} t(z)-a_{0} t^{*}(z)}{z}
$$

is of degree $n-1$ and has $r_{1}=r-1$ and $s_{1}=s$ number of zeros inside and on the unit circle $|z|=1$, respectively.

Lemma 2.2 (Schur-Cohn's algorithm [12, p. 383]). Given a polynomial $r(z)=$ $a_{0}+a_{1} z+\cdots+a_{n} z^{n}$ of degree $n$, let

$$
M_{k}=\left|\begin{array}{ll}
\bar{B}_{k}^{T} & A_{k} \\
\bar{A}_{k}^{T} & B_{k}
\end{array}\right| \quad(k=1,2 \ldots, n),
$$

where $A_{k}$ and $B_{k}$ are the triangular matrices

$$
A_{k}=\left(\begin{array}{cccc}
a_{0} & a_{1} & \ldots & a_{k-1} \\
& a_{0} & \ldots & a_{k-2} \\
& & \ddots & \vdots \\
& & & a_{0}
\end{array}\right) \quad \text { and } B_{k}=\left(\begin{array}{cccc}
\bar{a}_{n} & \bar{a}_{n-1} & \ldots & \bar{a}_{n-k+1} \\
& \bar{a}_{n} & \ldots & \bar{a}_{n-k+2} \\
& & \ddots & \vdots \\
& & & \bar{a}_{n}
\end{array}\right) \text {. }
$$

Then the polynomial $r$ has all its zeros inside the unit circle $|z|=1$ if and only if the determinants $M_{1}, M_{2}, \ldots, M_{n}$ are all positive.

Lemma 2.3 (Kumar et al. [7]). Let $f_{\beta}=h_{\beta}+\bar{g}_{\beta}$ be as in Theorem $D$ with dilatation $\omega=g_{\beta}^{\prime} / h_{\beta}^{\prime}$. Then $\widetilde{\omega}$, the dilatation of $F_{a} \widetilde{*} f_{\beta}$, is given by

$\widetilde{\omega}(z)=\left[\frac{2 \omega(1+\omega)\left(a+a z \cos \beta+z \cos \beta+z^{2}\right)-z \omega^{\prime}(1-a)\left(1+2 z \cos \beta+z^{2}\right)}{2\left(1+z \cos \beta+a z \cos \beta+a z^{2}\right)(1+\omega)-z \omega^{\prime}(1-a)\left(1+2 z \cos \beta+z^{2}\right)}\right]$.

We now proceed to state and prove our main result.

Theorem 2.4. Let $F_{a}=H_{a}+\bar{G}_{a}$ be given by (3) and let $f_{\pi / 2}=h_{\pi / 2}+\bar{g}_{\pi / 2}$ be the map obtained from (2) with $\beta=\pi / 2$ and dilatation

$$
\omega(z)=g_{\pi / 2}^{\prime}(z) / h_{\pi / 2}^{\prime}(z)=e^{i \theta} z^{n}(\theta \in \mathbb{R}, n \in \mathbb{N}) .
$$

Then $F_{a} \widetilde{*} f_{\pi / 2} \in S_{H}^{0}$ and is CHD for $a \in[(n-2) /(n+2), 1)$.

Proof. In view of Theorem B, we need only to show that $F_{a} \widetilde{*} f_{\pi / 2}$ is locally univalent and sense-preserving or equivalently, the dilatation $\widetilde{\omega}$ of $F_{a} \widetilde{*} f_{\pi / 2}$ satisfies $|\widetilde{\omega}(z)|<1$ for all $z \in E$. Setting $\omega(z)=e^{i \theta} z^{n}$ and $\beta=\pi / 2$ in (4), we get

(5)

$$
\begin{aligned}
& \widetilde{\omega}(z) \\
= & z^{n} e^{2 i \theta}\left[\frac{z^{n+2}+a z^{n}+\frac{1}{2}(2+a n-n) e^{-i \theta} z^{2}+\frac{1}{2}(2 a+a n-n) e^{-i \theta}}{\frac{1}{2}(2 a+a n-n) e^{i \theta} z^{n+2}+\frac{1}{2}(2+a n-n) e^{i \theta} z^{n}+a z^{2}+1}\right] \\
= & z^{n} e^{2 i \theta} \frac{p(z)}{p^{*}(z)}
\end{aligned}
$$


where

$$
p(z)=z^{n+2}+a z^{n}+\frac{1}{2}(2+a n-n) e^{-i \theta} z^{2}+\frac{1}{2}(2 a+a n-n) e^{-i \theta}
$$

and $p^{*}(z)=z^{n+2} \overline{p\left(\frac{1}{\bar{z}}\right)}$.

Obviously, if $z_{0}, z_{0} \neq 0$, is a zero of $p$, then $1 / \bar{z}_{0}$ is a zero of $p^{*}$. Hence, if $\alpha_{1}, \alpha_{2}, \ldots, \alpha_{n+2}$ are the zeros of $p$ (not necessarily distinct), then we can write

$$
\widetilde{\omega}(z)=z^{n} e^{2 i \theta} \frac{\left(z-\alpha_{1}\right)}{\left(1-\bar{\alpha}_{1} z\right)} \frac{\left(z-\alpha_{2}\right)}{\left(1-\bar{\alpha}_{2} z\right)} \cdots \frac{\left(z-\alpha_{n+2}\right)}{\left(1-\bar{\alpha}_{n+2} z\right)} .
$$

For $\left|\alpha_{i}\right| \leq 1$, since $\left(z-\alpha_{i}\right) /\left(1-\bar{\alpha}_{i} z\right)$ maps the closed unit disk onto itself, therefore to prove that $|\widetilde{\omega}|<1$ in $E$, we shall show that all the zeros of polynomial $p$, i.e., $\alpha_{1}, \alpha_{2}, \ldots, \alpha_{n+2}$ lie inside or on the unit circle $|z|=1$ for $a \in((n-2) /(n+2), 1) \backslash\{n /(n+2)\}$ (because, in the case when $a=$ $(n-2) /(n+2)$, we see from (5) that $|\widetilde{\omega}(z)|=\left|-z^{n} e^{i \theta}\right|<1$ and the case when $a=n /(n+2)$, where $z_{0}=0$ is a zero of $p$, will be taken up separately). To do this, we use Lemma 2.2 on the polynomial $p(z)=z^{n+2}+a z^{n}+$ $\frac{1}{2}(2+a n-n) e^{-i \theta} z^{2}+\frac{1}{2}(2 a+a n-n) e^{-i \theta}$ and show that all the determinants $M_{k}, k=1,2,3, \ldots, n+2$, are positive. Here, $M_{k}$ is obtained by comparing the polynomial $p$ with polynomial $r$ (with degree $n+2$ ) of Lemma 2.2 and is given by

$$
M_{k}=\left|\begin{array}{cc}
\bar{B}_{k}^{T} & A_{k} \\
\bar{A}_{k}^{T} & B_{k}
\end{array}\right| \quad(k=1,2,3, \ldots, n+2) .
$$

For entries of $A_{k}$ and $B_{k}$ we obviously have $a_{0}=\frac{1}{2}(2 a+a n-n) e^{-i \theta}, a_{1}=0$, $a_{2}=\frac{1}{2}(2+a n-n) e^{-i \theta}, a_{3}=0, \ldots, a_{n-1}=0, a_{n}=a, a_{n+1}=0$, and $a_{n+2}=1$.

Since $B_{k}$ is a non singular matrix, therefore,

$$
\left(\begin{array}{cc}
\bar{B}_{k}^{T} & A_{k} \\
\bar{A}_{k}^{T} & B_{k}
\end{array}\right)\left(\begin{array}{cc}
I & 0 \\
-B_{k}^{-1} \bar{A}_{k}^{T} & I
\end{array}\right)=\left(\begin{array}{cc}
\bar{B}_{k}^{T}-A_{k} B_{k}^{-1} \bar{A}_{k}^{T} & A_{k} \\
0 & B_{k}
\end{array}\right),
$$

which gives,

$$
M_{k}=\left|\begin{array}{cc}
\bar{B}_{k}^{T} & A_{k} \\
\bar{A}_{k}^{T} & B_{k}
\end{array}\right|=\left|\bar{B}_{k}^{T}-A_{k} B_{k}^{-1} \bar{A}_{k}^{T}\right| .
$$

For $n=1,2,3$ and 4, as the proof of our theorem follows from the results in [7] by substituting $\beta=\pi / 2$, so we take $n \geq 5$. We consider the following cases.

Case 1. When $1 \leq k \leq n$ and $n \geq 5$ is odd. In this case $A_{k}$ and $B_{k}$ are the following $k \times k$ matrices;

$$
A_{k}=\left(\begin{array}{ccccc}
a_{0} & a_{1} & a_{2} & \ldots & a_{k-1} \\
0 & a_{0} & a_{1} & \ldots & a_{k-2} \\
0 & 0 & a_{0} & \ldots & a_{k-3} \\
\vdots & \vdots & \vdots & \ddots & \vdots \\
0 & 0 & 0 & \ldots & a_{0}
\end{array}\right)=\left(\begin{array}{ccccc}
a_{0} & 0 & a_{2} & \ldots & 0 \\
0 & a_{0} & 0 & \ldots & 0 \\
0 & 0 & a_{0} & \ldots & 0 \\
\vdots & \vdots & \vdots & \ddots & \vdots \\
0 & 0 & 0 & \ldots & a_{0}
\end{array}\right) \text { and }
$$




$$
B_{k}=\left(\begin{array}{ccccc}
\bar{a}_{n+2} & \bar{a}_{n+1} & \bar{a}_{n} & \ldots & \bar{a}_{(n+2)-k+1} \\
0 & \bar{a}_{n+2} & \bar{a}_{n+1} & \ldots & \bar{a}_{(n+2)-k+2} \\
0 & 0 & \bar{a}_{n+2} & \ldots & \bar{a}_{(n+2)-k+3} \\
\vdots & \vdots & \vdots & \ddots & \vdots \\
0 & 0 & 0 & \ldots & \bar{a}_{n+2}
\end{array}\right)=\left(\begin{array}{ccccc}
1 & 0 & a & \ldots & 0 \\
0 & 1 & 0 & \ldots & 0 \\
0 & 0 & 1 & \ldots & 0 \\
\vdots & \vdots & \vdots & \ddots & \vdots \\
0 & 0 & 0 & \ldots & 1
\end{array}\right) .
$$

So, $\bar{B}_{k}^{T}-A_{k} B_{k}^{-1} \bar{A}_{k}^{T}=\left(C_{1} C_{2} C_{3} \cdots C_{k-2} C_{k-1} C_{k}\right)$, where $C_{i}, 1 \leq i \leq k$, are the following column matrices;

$$
C_{1}=\left(\begin{array}{c}
1-a_{0} \bar{a}_{0}-\bar{a}_{2}\left(-a a_{0}+a_{2}\right) \\
0 \\
a-a_{0} \bar{a}_{2} \\
\vdots \\
0 \\
0 \\
0
\end{array}\right), C_{2}=\left(\begin{array}{c}
0 \\
1-\bar{a}_{0} a_{0}-\bar{a}_{2}\left(-a a_{0}+a_{2}\right) \\
0 \\
\vdots \\
0 \\
0 \\
0
\end{array}\right),
$$

$C_{3}=\left(\begin{array}{c}-\bar{a}_{0}\left(-a a_{0}+a_{2}\right)+a \bar{a}_{2}\left(-a a_{0}+a_{2}\right) \\ 0 \\ 1-a_{0} \bar{a}_{0}-\bar{a}_{2}\left(-a a_{0}+a_{2}\right) \\ \vdots \\ 0 \\ 0 \\ 0\end{array}\right), C_{k-2}=\left(\begin{array}{c}(-a)^{\frac{k-5}{2}}\left[-\bar{a}_{0}\left(-a a_{0}+a_{2}\right)+a \bar{a}_{2}\left(-a a_{0}+a_{2}\right)\right] \\ 0 \\ (-a)^{\frac{k-7}{2}}\left[-\bar{a}_{0}\left(-a a_{0}+a_{2}\right)+a \bar{a}_{2}\left(-a a_{0}+a_{2}\right)\right] \\ \vdots \\ 1-a_{0} \bar{a}_{0}-\bar{a}_{2}\left(-a a_{0}+a_{2}\right) \\ 0 \\ a-a_{0} \bar{a}_{2}\end{array}\right)$,

$$
C_{k-1}=\left(\begin{array}{c}
(-a)^{\frac{k-5}{2}}\left[-\bar{a}_{0}\left(-a a_{0}+a_{2}\right)\right] \\
0 \\
\vdots \\
0 \\
1-a_{0} \bar{a}_{0} \\
0
\end{array}\right) \text { and } C_{k}=\left(\begin{array}{c}
(-a)^{\frac{k-3}{2}}\left(-\bar{a}_{0}\left(-a a_{0}+a_{2}\right)\right) \\
0 \\
(-a)^{\frac{k-5}{2}}\left(-\bar{a}_{0}\left(-a a_{0}+a_{2}\right)\right) \\
\vdots \\
\left(-\bar{a}_{0}\left(-a a_{0}+a_{2}\right)\right) \\
0 \\
1-a_{0} \bar{a}_{0}
\end{array}\right) \text {. }
$$

Now, by setting the values of coefficients $a_{0}, a_{2}$ from the polynomial $p$, we have
(a) $1-\bar{a}_{0} a_{0}-\bar{a}_{2}\left(-a a_{0}+a_{2}\right)=\frac{1}{4} n(2-n+2 a+a n)(1-a)(2-a)$,
(b) $-\bar{a}_{0}\left(-a a_{0}+a_{2}\right)+a \bar{a}_{2}\left(-a a_{0}+a_{2}\right)=\frac{1}{4} n(2-n+2 a+a n)(1-a)^{3}$,
(c) $-\bar{a}_{0}\left(-a a_{0}+a_{2}\right)=\frac{1}{4}(n-2 a-a n)(2-n+2 a+a n)(1-a)$,
(d) $a-a_{0} \bar{a}_{2}=\frac{1}{4} n(2-n+2 a+a n)(1-a)$,
(e) $1-a_{0} \bar{a}_{0}=\frac{1}{4}(n+2)(2-n+2 a+a n)(1-a)$.

If we write, $L_{r}=\left(\frac{1}{4}\right)^{r} n^{r-2}(2-n+2 a+a n)^{r}(1-a)^{r}, r \in \mathbb{N}$, then

$$
M_{k}=L_{k}\left|\begin{array}{cccccc}
(2-a) & 0 & (1-a)^{2} & \ldots & 0 & (-a)^{\frac{k-3}{2}}(n-2 a-a n) \\
0 & (2-a) & 0 & \ldots & (-a)^{\frac{k-5}{2}}(n-2 a-a n) & 0 \\
1 & 0 & (2-a) & \ldots & 0 & (-a)^{\frac{k-5}{2}}(n-2 a-a n) \\
\vdots & \vdots & \vdots & \ddots & \vdots & \vdots \\
0 & 0 & 0 & \ldots & (n+2) & 0 \\
0 & 0 & 0 & \ldots & 0 & (n+2)
\end{array}\right|
$$


ON HARMONIC CONVOLUTIONS INVOLVING A VERTICAL STRIP MAPPING 111

$$
\begin{aligned}
& =L_{k}\left|\begin{array}{cccccc}
(2-a) & 0 & (1-a)^{2} & \ldots & 0 & (-a)^{\frac{k-3}{2}}(n-2 a-a n) \\
0 & (2-a) & 0 & \ldots & (-a)^{\frac{k-5}{2}}(n-2 a-a n) & 0 \\
0 & 0 & \frac{3-2 a}{2-a} & \ldots & 0 & 2(-a)^{\frac{k-5}{2}} \frac{(n-2 a-a n)}{2-a} \\
\vdots & \vdots & \vdots & \ddots & \vdots & \vdots \\
0 & 0 & 0 & \ldots & \frac{n+k-1}{\frac{k-1}{2}-\frac{(k-3) a}{2}} & 0 \\
0 & 0 & 0 & \ldots & 0 & \frac{n+k+1}{\frac{k+1}{2}-\frac{(k-1) a}{2}}
\end{array}\right| \\
= & L_{k}(n+k-1)(n+k+1) .
\end{aligned}
$$

As $L_{k}$ is positive for $a \in((n-2) /(n+2), 1) \backslash\{n /(n+2)\}$, so $M_{k}$ is positive in this case.

Case 2. When $1 \leq k \leq n$ and $n \geq 5$ is even. In this case

$$
\begin{aligned}
& A_{k}=\left(\begin{array}{ccccc}
a_{0} & a_{1} & a_{2} & \ldots & a_{k-1} \\
0 & a_{0} & a_{1} & \ldots & a_{k-2} \\
0 & 0 & a_{0} & \ldots & a_{k-3} \\
\vdots & \vdots & \vdots & \ddots & \vdots \\
0 & 0 & 0 & \ldots & a_{0}
\end{array}\right)=\left(\begin{array}{ccccc}
a_{0} & 0 & a_{2} & \ldots & 0 \\
0 & a_{0} & 0 & \ldots & 0 \\
0 & 0 & a_{0} & \ldots & 0 \\
\vdots & \vdots & \vdots & \ddots & \vdots \\
0 & 0 & 0 & \ldots & a_{0}
\end{array}\right) \text { and } \\
& B_{k}=\left(\begin{array}{ccccc}
\bar{a}_{n+2} & \bar{a}_{n+1} & \bar{a}_{n} & \ldots & \bar{a}_{(n+2)-k+1} \\
0 & \bar{a}_{n+2} & \bar{a}_{n+1} & \ldots & \bar{a}_{(n+2)-k+2} \\
0 & 0 & \bar{a}_{n+2} & \ldots & \bar{a}_{(n+2)-k+3} \\
\vdots & \vdots & \vdots & \ddots & \vdots \\
0 & 0 & 0 & \ldots & \bar{a}_{n+2}
\end{array}\right)=\left(\begin{array}{ccccc}
1 & 0 & a & \ldots & 0 \\
0 & 1 & 0 & \ldots & 0 \\
0 & 0 & 1 & \ldots & 0 \\
\vdots & \vdots & \vdots & \ddots & \vdots \\
0 & 0 & 0 & \ldots & 1
\end{array}\right)
\end{aligned}
$$

so, $\bar{B}_{k}^{T}-A_{k} B_{k}^{-1} \bar{A}_{k}^{T}=\left(C_{1} C_{2} C_{3} \cdots C_{k-2} C_{k-1} C_{k}\right)$. Here

$$
C_{1}=\left(\begin{array}{c}
1-a_{0} \bar{a}_{0}-\bar{a}_{2}\left(-a a_{0}+a_{2}\right) \\
0 \\
a-a_{0} \bar{a}_{2} \\
\vdots \\
0 \\
0 \\
0
\end{array}\right), C_{2}=\left(\begin{array}{c}
0 \\
1-\bar{a}_{0} a_{0}-\bar{a}_{2}\left(-a a_{0}+a_{2}\right) \\
0 \\
\vdots \\
0 \\
0 \\
0
\end{array}\right)
$$$$
C_{3}=\left(\begin{array}{c}
-\bar{a}_{0}\left(-a a_{0}+a_{2}\right)+a \bar{a}_{2}\left(-a a_{0}+a_{2}\right) \\
0 \\
1-a_{0} \bar{a}_{0}-\bar{a}_{2}\left(-a a_{0}+a_{2}\right) \\
\vdots \\
0 \\
0
\end{array}\right), C_{k-2}=\left(\begin{array}{c}
0 \\
(-a)^{\frac{k-6}{2}}\left(-\bar{a}_{0}\left(-a a_{0}+a_{2}\right)+a \bar{a}_{2}\left(-a a_{0}+a_{2}\right)\right) \\
0 \\
\vdots \\
1-a_{0} \bar{a}_{0}-\bar{a}_{2}\left(-a a_{0}+a_{2}\right) \\
0 \\
a-a_{0} \bar{a}_{2}
\end{array}\right) \text {, }
$$

$$
C_{k-1}=\left(\begin{array}{c}
(-a)^{\frac{k-4}{2}}\left(-\bar{a}_{0}\left(-a a_{0}+a_{2}\right)\right) \\
0 \\
(-a)^{\frac{k-6}{2}}\left(-\bar{a}_{0}\left(-a a_{0}+a_{2}\right)\right) \\
\vdots \\
0 \\
1-a_{0} \bar{a}_{0} \\
0
\end{array}\right) \text { and } C_{k}=\left(\begin{array}{c}
(-a)^{\frac{k-4}{2}}\left(-\bar{a}_{0}\left(-a a_{0}+a_{2}\right)\right) \\
0 \\
\vdots \\
\left(-\bar{a}_{0}\left(-a a_{0}+a_{2}\right)\right) \\
0 \\
1-a_{0} \bar{a}_{0}
\end{array}\right) \text {. }
$$


Therefore,

$$
\begin{aligned}
& M_{k}=L_{k}\left|\begin{array}{cccccc}
(2-a) & 0 & (1-a)^{2} & \ldots & (-a)^{\frac{k-4}{2}}(n-2 a-a n) & 0 \\
0 & (2-a) & 0 & \ldots & 0 & (-a)^{\frac{k-4}{2}}(n-2 a-a n) \\
1 & 0 & (2-a) & \ldots & (-a)^{\frac{k-6}{2}}(n-2 a-a n) & 0 \\
\vdots & \vdots & \vdots & \ddots & \vdots & \vdots \\
0 & 0 & 0 & \ldots & (n+2) & 0 \\
0 & 0 & 0 & \ldots & 0 & (n+2) \\
(2-a) & 0 & (1-a)^{2} & \ldots & (-a)^{\frac{k-4}{2}}(n-2 a-a n) & 0 \\
0 & (2-a) & 0 & \ldots & 0 & (-a)^{\frac{k-4}{2}}(n-2 a-a n) \\
0 & 0 & \frac{3-2 a}{2-a} & \ldots & 2(-a)^{\frac{k-6}{2}} \frac{(n-2 a-a n)}{2-a} & 0 \\
\vdots & \vdots & \vdots & \ddots & \vdots & 0 \\
0 & 0 & 0 & \ldots & \frac{n+k}{\frac{(k-1)+1}{2}-\frac{(k-1)-1) a}{2}} & 0 \\
0 & 0 & 0 & \ldots & & \frac{n+k}{2}
\end{array}\right| \\
&=L_{k}(n+k)(n+k),
\end{aligned}
$$

which is positive for $a \in((n-2) /(n+2), 1) \backslash\{n /(n+2)\}$.

Case 3. When $k=n+1$ and $n$ is an odd positive integer. We have,

$$
\begin{aligned}
A_{n+1} & =\left(\begin{array}{ccccc}
a_{0} & a_{1} & a_{2} & \ldots & a_{n} \\
0 & a_{0} & a_{1} & \ldots & a_{n-1} \\
0 & 0 & a_{0} & \ldots & a_{n-2} \\
\vdots & \vdots & \vdots & \ddots & \vdots \\
0 & 0 & 0 & \ldots & a_{0}
\end{array}\right)=\left(\begin{array}{ccccc}
a_{0} & 0 & a_{2} & \ldots & a \\
0 & a_{0} & 0 & \ldots & 0 \\
0 & 0 & a_{0} & \ldots & 0 \\
\vdots & \vdots & \vdots & \ddots & \vdots \\
0 & 0 & 0 & \ldots & a_{0}
\end{array}\right) \text { and } \\
B_{n+1} & =\left(\begin{array}{ccccc}
\bar{a}_{n+2} & \bar{a}_{n+1} & \bar{a}_{n} & \ldots & \bar{a}_{2} \\
0 & \bar{a}_{n+2} & \bar{a}_{n+1} & \ldots & \bar{a}_{3} \\
0 & 0 & \bar{a}_{n+2} & \ldots & \bar{a}_{4} \\
\vdots & \vdots & \vdots & \ddots & \vdots \\
0 & 0 & 0 & \ldots & \bar{a}_{n+2}
\end{array}\right)=\left(\begin{array}{ccccc}
1 & 0 & a & \ldots & \bar{a}_{2} \\
0 & 1 & 0 & \ldots & 0 \\
0 & 0 & 1 & \ldots & 0 \\
\vdots & \vdots & \vdots & \ddots & \vdots \\
0 & 0 & 0 & \ldots & 1
\end{array}\right) .
\end{aligned}
$$

Thus, $\bar{B}_{n+1}^{T}-A_{n+1} B_{n+1}^{-1} \bar{A}_{n+1}^{T}=\left(C_{1} C_{2} C_{3} \cdots C_{n-1} C_{n} C_{n+1}\right)$, where

$$
\begin{aligned}
& C_{1}=\left(\begin{array}{c}
1-a_{0} \bar{a}_{0}-\bar{a}_{2}\left(-a a_{0}+a_{2}\right)-a\left(-a_{0} \bar{a}_{2}+a\right) \\
(-a)^{\frac{n-1}{2}}\left(-a a_{0}+a_{2}\right) \\
a-a_{0} \bar{a}_{2} \\
\vdots \\
-a\left(-a a_{0}+a_{2}\right) \\
0 \\
\left(-a a_{0}+a_{2}\right)
\end{array}\right), C_{2}=\left(\begin{array}{c}
1-a_{0} \bar{a}_{0}-\bar{a}_{2}\left(-a a_{0}+a_{2}\right) \\
0 \\
\vdots \\
0 \\
0 \\
0
\end{array}\right) \text {, } \\
& C_{3}=\left(\begin{array}{c}
-\bar{a}_{0}\left(-a a_{0}+a_{2}\right)+a \bar{a}_{2}\left(-a a_{0}+a_{2}\right) \\
0 \\
1-a_{0} \bar{a}_{0}-\bar{a}_{2}\left(-a a_{0}+a_{2}\right) \\
\vdots \\
0 \\
0 \\
0
\end{array}\right), C_{n-1}=\left(\begin{array}{c}
-\bar{a}_{2}\left(-a_{0} \bar{a}_{2}+a\right) \\
a^{\frac{n-5}{2}}\left[-\bar{a}_{0}\left(-a a_{0}+a_{2}\right)+a \bar{a}_{2}\left(-a a_{0}+a_{2}\right)\right] \\
0 \\
\vdots \\
1-a_{0} \bar{a}_{0}-\bar{a}_{2}\left(-a a_{0}+a_{2}\right) \\
0 \\
a-a_{0} \bar{a}_{2}
\end{array}\right) \text {, }
\end{aligned}
$$


ON HARMONIC CONVOLUTIONS INVOLVING A VERTICAL STRIP MAPPING

$$
C_{n}=\left(\begin{array}{c}
(-a)^{\frac{n-3}{2}}\left(-\bar{a}_{0}\left(-a a_{0}+a_{2}\right)\right) \\
0 \\
(-a)^{\frac{n-5}{2}}\left(-\bar{a}_{0}\left(-a a_{0}+a_{2}\right)\right) \\
\vdots \\
0 \\
1-a_{0} \bar{a}_{0} \\
0
\end{array}\right) \text { and } C_{n+1}=\left(\begin{array}{c}
-\bar{a}_{0}\left(-a_{0} \bar{a}_{2}+a\right) \\
(-a)^{\frac{n-3}{2}}\left[-\bar{a}_{0}\left(-a a_{0}+a_{2}\right)\right] \\
0 \\
\vdots \\
\left(-\bar{a}_{0}\left(-a a_{0}+a_{2}\right)\right) \\
0 \\
1-a_{0} \bar{a}_{0}
\end{array}\right)
$$

If $C_{j}$ is the $j^{\text {th }}$ column of $\bar{B}_{n+1}^{T}-A_{n+1} B_{n+1}^{-1} \bar{A}_{n+1}^{T}$, then the entries of $C_{j}(j=$ $2,3, \ldots, n-2)$ and $C_{n}$ are identical to those of the corresponding columns of $\bar{B}_{k}^{T}-A_{k} B_{k}^{-1} \bar{A}_{k}^{T}$ in Case 2. However, the entries of $C_{1}, C_{n-1}$, and $C_{n+1}$ are different. We split $C_{1}, C_{n-1}$ and $C_{n+1}$ in the following way:

$$
C_{1}=F_{1}+G_{1}+H_{1}, C_{n-1}=F_{n-1}+G_{n-1}, C_{n+1}=F_{n+1}+G_{n+1},
$$

where

$$
\begin{aligned}
F_{1}^{T}= & \left(1-a_{0} \bar{a}_{0}-\bar{a}_{2}\left(-a a_{0}+a_{2}\right), 0, a-a_{0} \bar{a}_{2}, 0, \ldots, 0\right), \\
G_{1}^{T}= & \left(0,(-a)^{\frac{n-1}{2}}\left(-a a_{0}+a_{2}\right), 0,(-a)^{\frac{n-3}{2}}\left(-a a_{0}+a_{2}\right), 0 \ldots,\left(-a a_{0}+a_{2}\right)\right), \\
H_{1}^{T}= & \left(-a\left(-a_{0} \bar{a}_{2}+a\right), 0,0, \ldots, 0\right) \\
F_{n-1}^{T}= & \left(0,(-a)^{\frac{n-5}{2}}\left[-\bar{a}_{0}\left(-a a_{0}+a_{2}\right)+a \bar{a}_{2}\left(-a a_{0}+a_{2}\right)\right], 0,\right. \\
& \left.(-a)^{\frac{n-7}{2}}\left[-\bar{a}_{0}\left(-a a_{0}+a_{2}\right)+a \bar{a}_{2}\left(-a a_{0}+a_{2}\right)\right], 0 \ldots, a-a_{0} \bar{a}_{2}\right), \\
G_{n-1}^{T}= & \left(-\bar{a}_{2}\left(-a_{0} \bar{a}_{2}+a\right), 0,0, \ldots, 0\right) ; \\
F_{n+1}^{T}= & \left(0,(-a)^{\frac{n-3}{2}}\left[-\bar{a}_{0}\left(-a a_{0}+a_{2}\right)\right], 0,(-a)^{\frac{n-3}{2}}\left[-\bar{a}_{0}\left(-a a_{0}+a_{2}\right)\right], 0,\right. \\
& \left.\cdots, 1-a_{0} \bar{a}_{0}\right) \text { and } \\
G_{n+1}^{T}= & \left(-\bar{a}_{0}\left(-a_{0} \bar{a}_{2}+a\right), 0,0, \ldots, 0\right) .
\end{aligned}
$$

Now$$
\left|\bar{B}_{n+1}^{T}-A_{n+1} B_{n+1}^{-1} \bar{A}_{n+1}^{T}\right|=\left|C_{1} C_{2} \cdots C_{n} C_{n+1}\right|
$$$$
=\left|F_{1} C_{2} \cdots F_{n-1} C_{n} F_{n+1}\right|+\left|G_{1} C_{2} \cdots F_{n-1} C_{n} F_{n+1}\right|+\left|H_{1} C_{2} \cdots F_{n-1} C_{n} F_{n+1}\right|
$$$$
+\left|F_{1} C_{2} \cdots F_{n-1} C_{n} G_{n+1}\right|+\left|G_{1} C_{2} \cdots F_{n-1} C_{n} G_{n+1}\right|+\left|H_{1} C_{2} \cdots F_{n-1} C_{n} G_{n+1}\right|
$$$$
+\left|F_{1} C_{2} \cdots G_{n-1} C_{n} F_{n+1}\right|+\left|G_{1} C_{2} \cdots G_{n-1} C_{n} F_{n+1}\right|+\left|H_{1} C_{2} \cdots G_{n-1} C_{n} F_{n+1}\right|
$$$$
+\left|F_{1} C_{2} \cdots G_{n-1} C_{n} G_{n+1}\right|+\left|G_{1} C_{2} \cdots G_{n-1} C_{n} G_{n+1}\right|+\left|H_{1} C_{2} \cdots G_{n-1} C_{n} G_{n+1}\right| \text {. }
$$

Out of these twelve determinants, only four, given in Table 1, are non-zero.

Adding all these determinants we get

$$
\begin{aligned}
M_{n+1} & =\left|\bar{B}_{n+1}^{T}-A_{n+1} B_{n+1}^{-1} \bar{A}_{n+1}^{T}\right| \\
& =L_{n+1} 8 n>0 \text { for } a \in((n-2) /(n+2), 1) \backslash\{n /(n+2)\} .
\end{aligned}
$$


Case 4. When $k=n+1$ and $n$ is an even positive integer. Here

$$
\begin{aligned}
A_{n+1} & =\left(\begin{array}{ccccc}
a_{0} & a_{1} & a_{2} & \ldots & a_{n} \\
0 & a_{0} & a_{1} & \ldots & a_{n-1} \\
0 & 0 & a_{0} & \ldots & a_{n-2} \\
\vdots & \vdots & \vdots & \ddots & \vdots \\
0 & 0 & 0 & \ldots & a_{0}
\end{array}\right)=\left(\begin{array}{ccccc}
a_{0} & 0 & a_{2} & \ldots & a \\
0 & a_{0} & 0 & \ldots & 0 \\
0 & 0 & a_{0} & \ldots & 0 \\
\vdots & \vdots & \vdots & \ddots & \vdots \\
0 & 0 & 0 & \ldots & a_{0}
\end{array}\right) \text { and } \\
B_{n+1} & =\left(\begin{array}{ccccc}
\bar{a}_{n+2} & \bar{a}_{n+1} & \bar{a}_{n} & \ldots & \bar{a}_{2} \\
0 & \bar{a}_{n+2} & \bar{a}_{n+1} & \ldots & \bar{a}_{3} \\
0 & 0 & \bar{a}_{n+2} & \ldots & \bar{a}_{4} \\
\vdots & \vdots & \vdots & \ddots & \vdots \\
0 & 0 & 0 & \ldots & \bar{a}_{n+2}
\end{array}\right)=\left(\begin{array}{ccccc}
1 & 0 & a & \ldots & \bar{a}_{2} \\
0 & 1 & 0 & \ldots & 0 \\
0 & 0 & 1 & \ldots & 0 \\
\vdots & \vdots & \vdots & \ddots & \vdots \\
0 & 0 & 0 & \ldots & 1
\end{array}\right) .
\end{aligned}
$$

One can verify that

$$
\bar{B}_{n+1}^{T}-A_{n+1} B_{n+1}^{-1} \bar{A}_{n+1}^{T}=\left(C_{1} C_{2} C_{3} \cdots C_{n-1} C_{n} C_{n+1}\right) .
$$

Here

$$
\begin{aligned}
& C_{1}=\left(\begin{array}{c}
1-a_{0} \bar{a}_{0}-\bar{a}_{2}\left(-a a_{0}+a_{2}\right)-a\left(-a_{0} \bar{a}_{2}+a\right)+(-a)^{\frac{n}{2}}\left(-a a_{0}+a_{2}\right) \\
0 \\
a-a_{0} \bar{a}_{2}+(-a)^{\frac{n-2}{2}}\left(-a a_{0}+a_{2}\right) \\
\vdots \\
-a\left(-a a_{0}+a_{2}\right) \\
0 \\
\left(-a a_{0}+a_{2}\right)
\end{array}\right), \\
& C_{2}=\left(\begin{array}{c}
0 \\
1-a_{0} \bar{a}_{0}-\bar{a}_{2}\left(-a a_{0}+a_{2}\right) \\
0 \\
\vdots \\
0 \\
0 \\
0
\end{array}\right), C_{3}=\left(\begin{array}{c}
-\bar{a}_{0}\left(-a a_{0}+a_{2}\right)+a \bar{a}_{2}\left(-a a_{0}+a_{2}\right) \\
0 \\
1-a_{0} \bar{a}_{0}-\bar{a}_{2}\left(-a a_{0}+a_{2}\right) \\
\vdots \\
0 \\
0 \\
0
\end{array}\right) \text {, } \\
& C_{n-1}=\left(\begin{array}{c}
a^{\frac{n-4}{2}}\left[-\bar{a}_{0}\left(-a a_{0}+a_{2}\right)+a \bar{a}_{2}\left(-a a_{0}+a_{2}\right)\right]-\bar{a}_{2}\left(-a_{0} \bar{a}_{2}+a\right) \\
0 \\
a^{\frac{n-6}{2}}\left[-\bar{a}_{0}\left(-a a_{0}+a_{2}\right)+a \bar{a}_{2}\left(-a a_{0}+a_{2}\right)\right] \\
\vdots \\
1-a_{0} \bar{a}_{0}-\bar{a}_{2}\left(-a a_{0}+a_{2}\right) \\
0 \\
a-a_{0} \bar{a}_{2}
\end{array}\right)
\end{aligned}
$$


ON HARMONIC CONVOLUTIONS INVOLVING A VERTICAL STRIP MAPPING

$C_{n}=\left(\begin{array}{c}0 \\ (-a)^{\frac{n-4}{2}}\left(-\bar{a}_{0}\left(-a a_{0}+a_{2}\right)\right) \\ 0 \\ \vdots \\ 0 \\ 1-a_{0} \bar{a}_{0} \\ 0\end{array}\right)$ and $C_{n+1}=\left(\begin{array}{c}(-a)^{\frac{n-2}{2}}\left(-\bar{a}_{0}\left(-a a_{0}+a_{2}\right)\right)-\bar{a}_{0}\left(-a_{0} \bar{a}_{2}+a\right) \\ 0 \\ (-a)^{\frac{n-4}{2}}\left(-\bar{a}_{0}\left(-a a_{0}+a_{2}\right)\right) \\ \vdots \\ \left(-\bar{a}_{0}\left(-a a_{0}+a_{2}\right)\right) \\ 0 \\ 1-a_{0} \bar{a}_{0}\end{array}\right)$.

We split $C_{1}, C_{n-1}$ and $C_{n+1}$ in the following way (entries in the remaining columns will be identical to those of the corresponding columns of $\bar{B}_{k}^{T}-$ $A_{k} B_{k}^{-1} \bar{A}_{k}^{T}$ in Case 1).

$$
C_{1}=F_{1}+G_{1}+H_{1}, C_{n-1}=F_{n-1}+G_{n-1}, C_{n+1}=F_{n+1}+G_{n+1},
$$

where

$$
\begin{aligned}
F_{1}^{T}= & \left(1-a_{0} \bar{a}_{0}-\bar{a}_{2}\left(-a a_{0}+a_{2}\right), 0, a-a_{0} \bar{a}_{2}, 0, \ldots, 0\right), \\
G_{1}^{T}= & \left((-a)^{\frac{n}{2}}\left(-a a_{0}+a_{2}\right), 0,(-a)^{\frac{n-2}{2}}\left(-a a_{0}+a_{2}\right), 0 \ldots,\left(-a a_{0}+a_{2}\right)\right), \\
H_{1}^{T}= & \left(-a\left(-a_{0} \bar{a}_{2}+a\right), 0,0, \ldots, 0\right) \\
F_{n-1}^{T}= & \left((-a)^{\frac{n-4}{2}}\left[-\bar{a}_{0}\left(-a a_{0}+a_{2}\right)+a \bar{a}_{2}\left(-a a_{0}+a_{2}\right)\right], 0,\right. \\
& (-a)^{\frac{n-6}{2}}\left[-\bar{a}_{0}\left(-a a_{0}+a_{2}\right)\right. \\
& \left.\left.+a \bar{a}_{2}\left(-a a_{0}+a_{2}\right)\right], 0 \ldots, a-a_{0} \bar{a}_{2}\right), \\
G_{n-1}^{T}= & \left(-\bar{a}_{2}\left(-a_{0} \bar{a}_{2}+a\right), 0,0, \ldots, 0\right) ; \\
F_{n+1}^{T}= & \left((-a)^{\frac{n-2}{2}}\left[-\bar{a}_{0}\left(-a a_{0}+a_{2}\right)\right], 0,(-a)^{\frac{n-4}{2}}\left[-\bar{a}_{0}\left(-a a_{0}+a_{2}\right)\right], 0,\right. \\
& \left.\cdots, 1-a_{0} \bar{a}_{0}\right) \text { and } \\
G_{n+1}^{T}= & \left(-\bar{a}_{0}\left(-a_{0} \bar{a}_{2}+a\right), 0,0, \ldots, 0\right) .
\end{aligned}
$$

As in Case 3,

$$
\begin{aligned}
& M_{n+1}=\left|C_{1} C_{2} \cdots C_{n} C_{n+1}\right| \\
= & \left|F_{1} C_{2} \cdots F_{n-1} C_{n} F_{n+1}\right|+\left|G_{1} C_{2} \cdots F_{n-1} C_{n} F_{n+1}\right|+\left|H_{1} C_{2} \cdots F_{n-1} C_{n} F_{n+1}\right| \\
& +\left|F_{1} C_{2} \cdots F_{n-1} C_{n} G_{n+1}\right|+\left|G_{1} C_{2} \cdots F_{n-1} C_{n} G_{n+1}\right|+\left|H_{1} C_{2} \cdots F_{n-1} C_{n} G_{n+1}\right| \\
& +\left|F_{1} C_{2} \cdots G_{n-1} C_{n} F_{n+1}\right|+\left|G_{1} C_{2} \cdots G_{n-1} C_{n} F_{n+1}\right|+\left|H_{1} C_{2} \cdots G_{n-1} C_{n} F_{n+1}\right| \\
& +\left|F_{1} C_{2} \cdots G_{n-1} C_{n} G_{n+1}\right|+\left|G_{1} C_{2} \cdots G_{n-1} C_{n} G_{n+1}\right|+\left|H_{1} C_{2} \cdots G_{n-1} C_{n} G_{n+1}\right| .
\end{aligned}
$$

Out of these twelve determinants, only seven given in Table 2, are non-zero. Adding values of all these determinants we see that, when $n / 2$ is odd:

$$
M_{n+1}=L_{n+1} 8 n(1-\cos \theta)>0 \quad \text { for } \theta \neq 2 m \pi, m \in \mathbb{Z} \text { and }
$$

when $n / 2$ is even:

$$
M_{n+1}=L_{n+1} 8 n(1+\cos \theta)>0 \quad \text { for } \theta \neq(2 m+1) \pi, m \in \mathbb{Z} .
$$


Cases for $\theta=2 m \pi$ and $\theta=(2 m+1) \pi$ will be considered separately in the later part of this proof.

Case 5. When $k=n+2$ and $n$ is odd. In this case,

$$
\bar{B}_{n+2}^{T}-A_{n+2} B_{n+2}^{-1} \bar{A}_{n+2}^{T}=\left(C_{1} C_{2} C_{3} \cdots C_{n-1} C_{n} C_{n+1} C_{n+2}\right) .
$$

Here

$$
C_{1}=\left(\begin{array}{c}
1-a_{0} \bar{a}_{0}-\bar{a}_{2}\left(-a a_{0}+a_{2}\right)-a\left(-a_{0} \bar{a}_{2}+a\right) \\
(-a)^{\frac{n-1}{2}}\left(-a a_{0}+a_{2}\right) \\
a-a_{0} \bar{a}_{2} \\
(-a)^{\frac{n-3}{2}}\left(-a a_{0}+a_{2}\right) \\
\vdots \\
0 \\
\left(-a a_{0}+a_{2}\right) \\
0
\end{array}\right), C_{2}=\left(\begin{array}{c}
(-a)^{\frac{n+1}{2}}\left(-a a_{0}+a_{2}\right) \\
1-a_{0} \bar{a}_{0}-\bar{a}_{2}\left(-a a_{0}+a_{2}\right)-a\left(-a_{0} \bar{a}_{2}+a\right) \\
(-a)^{\frac{n-1}{2}}\left(-a a_{0}+a_{2}\right) \\
a-a a_{2} \\
\vdots \\
-a\left(-a a_{0}+a_{2}\right) \\
0 \\
\left(-a a_{0}+a_{2}\right)
\end{array}\right)
$$$$
C_{3}=\left(\begin{array}{c}
-\bar{a}_{0}\left(-a a_{0}+a_{2}\right)+a \bar{a}_{2}\left(-a a_{0}+a_{2}\right) \\
0 \\
1-a_{0} \bar{a}_{0}-\bar{a}_{2}\left(-a a_{0}+a_{2}\right) \\
0 \\
\vdots \\
0 \\
0
\end{array}\right), C_{n-1}=\left(\begin{array}{c}
-\bar{a}_{2}\left(-a_{0} \bar{a}_{2}+a\right) \\
(-a)^{\frac{n-5}{2}}\left(-\bar{a}_{0}\left(-a a_{0}+a_{2}\right)+a \bar{a}_{2}\left(-a a_{0}+a_{2}\right)\right) \\
0 \\
(-a)^{\frac{n-7}{2}}\left(-\bar{a}_{0}\left(-a a_{0}+a_{2}\right)+a \bar{a}_{2}\left(-a a_{0}+a_{2}\right)\right) \\
\vdots \\
0 \\
a-a_{0} \bar{a}_{2} \\
0
\end{array}\right),
$$$$
C_{n}=\left(\begin{array}{c}
a^{\frac{n-3}{2}}\left[-\bar{a}_{0}\left(-a a_{0}+a_{2}\right)+a \bar{a}_{2}\left(-a a_{0}+a_{2}\right)\right] \\
-\bar{a}_{2}\left(-a_{0} \bar{a}_{2}+a\right) \\
a^{\frac{n-5}{2}}\left[-\bar{a}_{0}\left(-a a_{0}+a_{2}\right)+a \bar{a}_{2}\left(-a a_{0}+a_{2}\right)\right] \\
0 \\
\vdots \\
1-a_{0} \bar{a}_{0}-\bar{a}_{2}\left(-a a_{0}+a_{2}\right) \\
0 \\
a-a_{0} \bar{a}_{2}
\end{array}\right), C_{n+1}=\left(\begin{array}{c}
-\bar{a}_{0}\left(-a_{0} \bar{a}_{2}+a\right) \\
(-a)^{\frac{n-3}{2}}\left(-\bar{a}_{0}\left(-a a_{0}+a_{2}\right)\right) \\
0 \\
(-a)^{\frac{n-5}{2}}\left(-\bar{a}_{0}\left(-a a_{0}+a_{2}\right)\right) \\
\vdots \\
0 \\
1-a_{0} \bar{a}_{0} \\
0
\end{array}\right) \text { and }
$$

$$
C_{n+2}=\left(\begin{array}{c}
(-a)^{\frac{n-1}{2}}\left(-\bar{a}_{0}\left(-a a_{0}+a_{2}\right)\right) \\
-\bar{a}_{0}\left(-a_{0} \bar{a}_{2}+a\right) \\
(-a)^{\frac{n-3}{2}}\left(-\bar{a}_{0}\left(-a a_{0}+a_{2}\right)\right) \\
0 \\
\vdots \\
\left(-\bar{a}_{0}\left(-a a_{0}+a_{2}\right)\right) \\
0 \\
1-a_{0} \bar{a}_{0}
\end{array}\right) .
$$

If $C_{j}(j=1,2, \ldots, n+2)$ are the columns of $\bar{B}_{n+2}^{T}-A_{n+2} B_{n+2}^{-1} \bar{A}_{n+2}^{T}$, then we split $C_{1}, C_{2}, C_{n-1}, C_{n}, C_{n+1}$ and $C_{n+2}$ as under (entries in the remaining columns will be identical to those of the corresponding columns of $\bar{B}_{k}^{T}-$ $A_{k} B_{k}^{-1} \bar{A}_{k}^{T}$ in Case 1).

$$
\begin{aligned}
& C_{1}=F_{1}+G_{1}+H_{1}, C_{2}=F_{2}+G_{2}+H_{2}, C_{n-1}=F_{n-1}+G_{n-1}, \\
& C_{n}=F_{n}+G_{n}, C_{n+1}=F_{n+1}+G_{n+1} \text { and } C_{n+2}=F_{n+2}+G_{n+2},
\end{aligned}
$$

where,

$$
\begin{aligned}
& F_{1}^{T}=\left(1-a_{0} \bar{a}_{0}-\bar{a}_{2}\left(-a a_{0}+a_{2}\right), 0, a-a_{0} \bar{a}_{2}, 0, \ldots, 0\right), \\
& G_{1}^{T}=\left(0,(-a)^{\frac{n-1}{2}}\left(-a a_{0}+a_{2}\right), 0,(-a)^{\frac{n-3}{2}}\left(-a a_{0}+a_{2}\right), 0 \ldots,\left(-a a_{0}+a_{2}\right), 0\right),
\end{aligned}
$$




$$
\begin{aligned}
H_{1}^{T}= & \left(-a\left(-a_{0} \bar{a}_{2}+a\right), 0,0, \ldots, 0\right) ; \\
F_{2}^{T}= & \left(0,1-a_{0} \bar{a}_{0}-\bar{a}_{2}\left(-a a_{0}+a_{2}\right), 0, a-a_{0} \bar{a}_{2}, 0, \ldots, 0\right), \\
G_{2}^{T}= & \left((-a)^{\frac{n+1}{2}}\left(-a a_{0}+a_{2}\right), 0,(-a)^{\frac{n-1}{2}}\left(-a a_{0}+a_{2}\right), 0,\right. \\
& \left.(-a)^{\frac{n-3}{2}}\left(-a a_{0}+a_{2}\right), 0 \ldots,\left(-a a_{0}+a_{2}\right)\right), \\
H_{2}^{T}= & \left(0,-a\left(-a_{0} \bar{a}_{2}+a\right), 0,0, \ldots, 0\right) ; \\
F_{n-1}^{T}= & \left(0,(-a)^{\frac{n-5}{2}}\left[-\bar{a}_{0}\left(-a a_{0}+a_{2}\right)+a \bar{a}_{2}\left(-a a_{0}+a_{2}\right)\right], 0,\right. \\
& \left.(-a)^{\frac{n-7}{2}}\left[-\bar{a}_{0}\left(-a a_{0}+a_{2}\right)+a \bar{a}_{2}\left(-a a_{0}+a_{2}\right)\right], 0 \ldots, a-a_{0} \bar{a}_{2}, 0\right), \\
G_{n-1}^{T}= & \left(-\bar{a}_{2}\left(-a_{0} \bar{a}_{2}+a\right), 0,0, \ldots, 0\right) ; \\
F_{n}^{T}= & \left((-a)^{\frac{n-3}{2}}\left[-\bar{a}_{0}\left(-a a_{0}+a_{2}\right)+a \bar{a}_{2}\left(-a a_{0}+a_{2}\right)\right], 0,\right. \\
& \left.(-a)^{\frac{n-5}{2}}\left[-\bar{a}_{0}\left(-a a_{0}+a_{2}\right)+a \bar{a}_{2}\left(-a a_{0}+a_{2}\right)\right], 0 \ldots, a-a_{0} \bar{a}_{2}\right), \\
G_{n}^{T}= & \left(0,-\bar{a}_{2}\left(-a_{0} \bar{a}_{2}+a\right), 0,0, \ldots, 0\right) ; \\
F_{n+1}^{T}= & \left(0,(-a)^{\frac{n-3}{2}}\left[-\bar{a}_{0}\left(-a a_{0}+a_{2}\right)\right], 0,(-a)^{\frac{n-5}{2}}\left[-\bar{a}_{0}\left(-a a_{0}+a_{2}\right)\right], 0,\right. \\
& \left.\ldots, 1-a_{0} \bar{a}_{0}, 0\right), \\
G_{n+1}^{T}= & \left(-\bar{a}_{0}\left(-a_{0} \bar{a}_{2}+a\right), 0,0, \ldots, 0\right) \text { and } \\
F_{n+2}^{T}= & \left((-a)^{\frac{n-1}{2}}\left[-\bar{a}_{0}\left(-a a_{0}+a_{2}\right)\right], 0,(-a)^{\frac{n-3}{2}}\left[-\bar{a}_{0}\left(-a a_{0}+a_{2}\right)\right], 0,\right. \\
& (-a)^{\frac{n-5}{2}}\left[-\bar{a}_{0}\left(-a a_{0}+a_{2}\right), 0, \ldots, 1-a_{0} \bar{a}_{0}\right), \\
G_{n+2}^{T}= & \left(0,-\bar{a}_{0}\left(-a_{0} \bar{a}_{2}+a\right), 0,0, \ldots, 0\right) .
\end{aligned}
$$

As a consequence of above splitting of columns, $M_{n+2}$ can be written as a sum of 144 determinants out of which 123 vanish and values of remaining 21 non-zero determinants are listed in Table 3. Adding all these determinants we get

$$
\begin{aligned}
M_{n+2} & =\left|\bar{B}_{n+2}^{T}-A_{n+2} B_{n+2}^{-1} \bar{A}_{n+2}^{T}\right| \\
& =L_{n+2}[8(1+\cos 2 \theta)]>0 \text { for } \theta \neq(2 m+1) \frac{\pi}{2}, m \in \mathbb{Z} .
\end{aligned}
$$

The case when $\theta=(2 m+1) \frac{\pi}{2}$ will be considered separately.

Case 6. When $k=n+2$ and $n$ is even. In this case,

$$
\bar{B}_{n+2}^{T}-A_{n+2} B_{n+2}^{-1} \bar{A}_{n+2}^{T}=\left(C_{1} C_{2} C_{3} \cdots C_{n-1} C_{n} C_{n+1} C_{n+2}\right) .
$$

Here

$$
\begin{aligned}
C_{1}^{T}= & \left(1-a_{0} \bar{a}_{0}-\bar{a}_{2}\left(-a a_{0}+a_{2}\right)-a\left(-a_{0} \bar{a}_{2}+a\right)+(-a)^{\frac{n}{2}}\left(-a a_{0}+a_{2}\right), 0,\right. \\
& \left(a-a_{0} \bar{a}_{2}+(-a)^{\frac{n-2}{2}}\left(-a a_{0}+a_{2}\right), 0, \ldots, 0,\left(-a a_{0}+a_{2}\right), 0\right) ; \\
C_{2}^{T}= & \left(1-a_{0} \bar{a}_{0}-\bar{a}_{2}\left(-a a_{0}+a_{2}\right)-a\left(-a_{0} \bar{a}_{2}+a\right)+(-a)^{\frac{n}{2}}\left(-a a_{0}+a_{2}\right), 0,\right. \\
& \left.a-a_{0} \bar{a}_{2}+(-a)^{\frac{n-2}{2}}\left(-a a_{0}+a_{2}\right), \ldots,-a\left(-a a_{0}+a_{2}\right), 0,\left(-a a_{0}+a_{2}\right)\right) ;
\end{aligned}
$$




$$
\begin{aligned}
C_{3}^{T}= & \left(-\bar{a}_{0}\left(-a a_{0}+a_{2}\right)+a \bar{a}_{2}\left(-a a_{0}+a_{2}\right), 0,1-a_{0} \bar{a}_{0}-\bar{a}_{2}\left(-a a_{0}+a_{2}\right), 0,\right. \\
& \ldots, 0,0) ; \\
C_{n-1}^{T}= & \left((-a)^{\frac{n-4}{2}}\left[-\bar{a}_{0}\left(-a a_{0}+a_{2}\right)+a \bar{a}_{2}\left(-a a_{0}+a_{2}\right)\right]-\bar{a}_{2}\left(-a_{0} \bar{a}_{2}+a\right), 0,\right. \\
& \left.(-a)^{\frac{n-6}{2}}\left[-\bar{a}_{0}\left(-a a_{0}+a_{2}\right)+a \bar{a}_{2}\left(-a a_{0}+a_{2}\right)\right], 0, \ldots, 0, a-a_{0} \bar{a}_{2}, 0\right) ; \\
C_{n}^{T}= & \left(0,(-a)^{\frac{n-4}{2}}\left[-\bar{a}_{0}\left(-a a_{0}+a_{2}\right)+a \bar{a}_{2}\left(-a a_{0}+a_{2}\right)\right]-\bar{a}_{2}\left(-a_{0} \bar{a}_{2}+a\right), 0,\right. \\
& (-a)^{\frac{n-6}{2}}\left[-\bar{a}_{0}\left(-a a_{0}+a_{2}\right)+a \bar{a}_{2}\left(-a a_{0}+a_{2}\right)\right], \ldots, \\
& \left.1-a_{0} \bar{a}_{0}-\bar{a}_{2}\left(-a a_{0}+a_{2}\right), 0, a-a_{0} \bar{a}_{2}\right) ; \\
C_{n+1}^{T}= & \left((-a)^{\frac{n-2}{2}}\left[-\bar{a}_{0}\left(-a a_{0}+a_{2}\right)\right]-\bar{a}_{0}\left(-a_{0} \bar{a}_{2}+a\right), 0,\right. \\
& \left.(-a)^{\frac{n-4}{2}}\left[-\bar{a}_{0}\left(-a a_{0}+a_{2}\right)\right], 0, \ldots, 0,1-a_{0} \bar{a}_{0}, 0 .\right) \text { and } \\
C_{n+2}^{T}= & \left(0,(-a)^{\frac{n-2}{2}}\left[-\bar{a}_{0}\left(-a a_{0}+a_{2}\right)\right]-\bar{a}_{0}\left(-a_{0} \bar{a}_{2}+a\right), 0,\right. \\
& \left.(-a)^{\frac{n-4}{2}}\left[-\bar{a}_{0}\left(-a a_{0}+a_{2}\right)\right], \ldots,\left[-\bar{a}_{0}\left(-a a_{0}+a_{2}\right)\right], 0,1-a_{0} \bar{a}_{0}\right) .
\end{aligned}
$$

As in the last case, here we split the columns $C_{1}, C_{2}, C_{n-1}, C_{n}, C_{n+1}$ and $C_{n+2}$ as $C_{1}=F_{1}+G_{1}+H_{1}, C_{2}=F_{2}+G_{2}+H_{2}, C_{n-1}=F_{n-1}+G_{n-1}, C_{n}=$ $F_{n}+G_{n}, C_{n+1}=F_{n+1}+G_{n+1}$ and $C_{n+2}=F_{n+2}+G_{n+2}$, where

$$
\begin{aligned}
F_{1}^{T}= & \left(1-a_{0} \bar{a}_{0}-\bar{a}_{2}\left(-a a_{0}+a_{2}\right), 0, a-a_{0} \bar{a}_{2}, 0, \ldots, 0\right), \\
G_{1}^{T}= & \left((-a)^{\frac{n}{2}}\left(-a a_{0}+a_{2}\right), 0,(-a)^{\frac{n-2}{2}}\left(-a a_{0}+a_{2}\right), 0 \ldots,\left(-a a_{0}+a_{2}\right), 0\right), \\
H_{1}^{T}= & \left(-a\left(-a_{0} \bar{a}_{2}+a\right), 0,0, \ldots, 0\right) ; \\
F_{2}^{T}= & \left(0,1-a_{0} \bar{a}_{0}-\bar{a}_{2}\left(-a a_{0}+a_{2}\right), 0, a-a_{0} \bar{a}_{2}, 0, \ldots, 0\right), \\
G_{2}^{T}= & \left(0,(-a)^{\frac{n}{2}}\left(-a a_{0}+a_{2}\right), 0,(-a)^{\frac{n-1}{2}}\left(-a a_{0}+a_{2}\right), 0,\right. \\
& \left.(-a)^{\frac{n-2}{2}}\left(-a a_{0}+a_{2}\right), 0, \ldots,\left(-a a_{0}+a_{2}\right)\right), \\
H_{2}^{T}= & \left(0,-a\left(-a_{0} \bar{a}_{2}+a\right), 0,0, \ldots, 0\right) ; \\
F_{n-1}^{T}= & \left((-a)^{\frac{n-4}{2}}\left[-\bar{a}_{0}\left(-a a_{0}+a_{2}\right)+a \bar{a}_{2}\left(-a a_{0}+a_{2}\right)\right], 0,\right. \\
& \left.(-a)^{\frac{n-6}{2}}\left[-\bar{a}_{0}\left(-a a_{0}+a_{2}\right)+a \bar{a}_{2}\left(-a a_{0}+a_{2}\right)\right], 0 \ldots, a-a_{0} \bar{a}_{2}, 0\right), \\
G_{n-1}^{T}= & \left(-\bar{a}_{2}\left(-a_{0} \bar{a}_{2}+a\right), 0,0, \ldots\right) ; \\
F_{n}^{T}= & \left(0,(-a)^{\frac{n-4}{2}}\left[-\bar{a}_{0}\left(-a a_{0}+a_{2}\right)+a \bar{a}_{2}\left(-a a_{0}+a_{2}\right)\right], 0,\right. \\
& \left.(-a)^{\frac{n-6}{2}}\left[-\bar{a}_{0}\left(-a a_{0}+a_{2}\right)+a \bar{a}_{2}\left(-a a_{0}+a_{2}\right)\right], 0 \ldots, a-a_{0} \bar{a}_{2}\right), \\
G_{n}^{T}= & \left(0,-\bar{a}_{2}\left(-a_{0} \bar{a}_{2}+a\right), 0,0, \ldots\right) ; \\
F_{n+1}^{T}= & \left((-a)^{\frac{n-2}{2}}\left[-\bar{a}_{0}\left(-a a_{0}+a_{2}\right)\right], 0,(-a)^{\frac{n-4}{2}}\left[-\bar{a}_{0}\left(-a a_{0}+a_{2}\right)\right], 0,\right. \\
& \left.\ldots, 1-a_{0} \bar{a}_{0}, 0\right), \\
G_{n+1}^{T}= & \left(-\bar{a}_{0}\left(-a_{0} \bar{a}_{2}+a\right), 0,0, \ldots, 0\right) \text { and }
\end{aligned}
$$




$$
\begin{aligned}
F_{n+2}^{T}= & \left(0,(-a)^{\frac{n-2}{2}}\left[-\bar{a}_{0}\left(-a a_{0}+a_{2}\right)\right], 0,(-a)^{\frac{n-4}{2}}\left[-\bar{a}_{0}\left(-a a_{0}+a_{2}\right)\right], 0,\right. \\
& \left.(-a)^{\frac{n-6}{2}}\left[-\bar{a}_{0}\left(-a a_{0}+a_{2}\right)\right], 0, \ldots, 1-a_{0} \bar{a}_{0}\right), \\
G_{n+2}^{T}= & \left(0,-\bar{a}_{0}\left(-a_{0} \bar{a}_{2}+a\right), 0,0, \ldots, 0\right) .
\end{aligned}
$$

In this case, $M_{n+2}$ is a sum of 144 determinants out of which only 49, listed in Table 4 , are non-zero. Adding values of all these determinants we obtain that, when $n / 2$ is odd:

$$
\begin{aligned}
M_{n+2} & =\left|\bar{B}_{n+2}^{T}-A_{n+2} B_{n+2}^{-1} \bar{A}_{n+2}^{T}\right| \\
& =L_{n+2}\left[16(1-\cos \theta)^{2}\right]>0 \quad \text { for } \theta \neq 2 m \pi, m \in \mathbb{Z} \text { and }
\end{aligned}
$$

when $n / 2$ is even:

$$
M_{n+2}=L_{n+2}\left[16(1+\cos \theta)^{2}\right]>0 \quad \text { for } \theta \neq(2 m+1) \pi, m \in \mathbb{Z} .
$$

Now we settle the particular cases. First consider the case when $k=n+1$ or $k=n+2$ and $n$ is even.

1. When $\theta=(2 m+1) \pi, m \in \mathbb{Z}$, and $n / 2$ is also even. In this case the polynomial $p$ given in (6) reduces to:

$$
\begin{aligned}
p(z)= & z^{n+2}+a z^{n}-\frac{1}{2}(2+a n-n) z^{2}-\frac{1}{2}(2 a+a n-n) \\
= & \left(z^{2}+1\right)\left[z^{n}+(a-1) z^{n-2}-(a-1) z^{n-4}+\cdots+(a-1) z^{2}\right. \\
& \left.-\frac{1}{2}(2 a+a n-n)\right] \\
= & \left(z^{2}+1\right) q(z) .
\end{aligned}
$$

It suffices to show that zeros of $q$ lie inside or on $|z|=1$. Since $\left|\frac{1}{2}(2 a+a n-n)\right|<$ 1 , whenever $a \in((n-2) /(n+2), 1) \backslash\{n /(n+2)\}$, by applying Lemma 2.1 on $q$, after comparing it with $t$, we get

$$
\begin{aligned}
& q_{1}(z) \\
= & \frac{\bar{a}_{n} q(z)-a_{0} q^{*}(z)}{z} \\
= & (1-a)\left(1+\frac{1}{2}(2 a+a n-n)\right) z\left\{\left(1+\frac{n}{2}\right) z^{n-2}-z^{n-4}+z^{n-6}-\cdots+z^{2}-1\right\} \\
= & (1-a)\left(1+\frac{1}{2}(2 a+a n-n)\right) z r_{1}(z),
\end{aligned}
$$

where, $r_{1}(z)=\left(1+\frac{n}{2}\right) z^{n-2}-z^{n-4}+z^{n-6}-\cdots+z^{2}-1$.

The number of zeros of $q_{1}$ inside the unit circle is one less than the number of zeros of $q$ inside the unit circle and so the number of zeros of $r_{1}$ inside the unit circle is two less than the number of zeros of $q$ inside the unit circle. As $1<1+n / 2$, therefore by applying Lemma 2.1 again on $r_{1}$ we get

$$
q_{2}(z)=z \frac{n}{2}\left[\left(2+\frac{n}{2}\right) z^{n-4}-z^{n-6}+z^{n-8}-\cdots-z^{2}+1\right]=z r_{2}(z) .
$$


The number of zeros of $r_{2}$ inside the circle $|z|=1$ is four less than the number of zeros of $q$ inside the unit circle. Similarly using Lemma 2.1 on $r_{2}$ we have,

$$
q_{3}(z)=z\left(1+\frac{n}{2}\right)\left[\left(3+\frac{n}{2}\right) z^{n-6}-z^{n-8}+z^{n-10}-\cdots+z^{2}-1\right] .
$$

Continuing in this manner we derive that

$$
\begin{aligned}
& q_{\lambda}(z) \\
= & z\left[(\lambda-2)+\frac{n}{2}\right]\left\{\left(\lambda+\frac{n}{2}\right) z^{n-2 \lambda}-z^{n-2(\lambda+1)}+\cdots+(-1)^{\lambda+1} z^{2}+(-1)^{\lambda}\right\} \\
= & z r_{\lambda}(z), \lambda=2,3, \ldots,(n / 2-1) .
\end{aligned}
$$

The number of zeros of $r_{\lambda}$ inside the unit circle is $2 \lambda$ less than the number of zeros of $q$ inside the unit circle and in particular, for $\lambda=(n / 2-1)$,

$$
q_{n / 2-1}(z)=(n-3)\left\{(n-1) z^{2}-1\right\},
$$

which has $(n-2)$ less number of zeros inside the unit circle than the number of zeros of $q$ inside the unit circle. But the zeros of $q_{n / 2-1}$ are $\pm(n-1)^{-1 / 2}$ which lie inside the unit circle $|z|=1$. Consequently, all the zeros of $q$ lie inside $|z|=1$.

2. When $\theta=2 m \pi, m \in \mathbb{Z}$, and $n / 2$ is odd. In this case the polynomial

$$
p(z)=\left(z^{2}+1\right) \eta(z)
$$

where

$\eta(z)=\left(z^{2}+1\right)\left[z^{n}+(a-1) z^{n-2}-(a-1) z^{n-4}+\cdots-(a-1) z^{2}+\frac{1}{2}(2 a+a n-n)\right]$.

Proceeding on the same lines as above we see that all the zeros of $p$ lie inside or on the circle $|z|=1$.

Next we consider the case when $k=n+2, n$ is odd and $\theta=(2 m+1) \pi / 2$, $m \in \mathbb{Z}$.

(i) If $n=4 u+1, u=1,2,3, \ldots$; in this case $p(z)=(z+i) \zeta(z)$ and $\zeta(z)=$ $z^{n+1}-i z^{n}+(a-1) z^{n-1}-i(a-1) z^{n-2}-(a-1) z^{n-3}+\cdots+\frac{i}{2}(2 a+a n-n)+$ $\frac{1}{2}(2 a+a n-n)$.

(ii) If $n=4 u-1, u=2,3,4, \ldots$; in this case $p(z)=(z-i) \xi(z)$, where $\xi(z)=z^{n+1}+i z^{n}+(a-1) z^{n-1}+i(a-1) z^{n-2}-(a-1) z^{n-3}-i(a-1) z^{n-4}+$ $\cdots+\frac{i}{2}(2 a+a n-n)-\frac{1}{2}(2 a+a n-n)$.

Again proceeding as above we conclude that all the zeros of $p$ lie inside or on the circle $|z|=1$.

Lastly, we take up the case when $a=n /(n+2)$. In this case

$$
\widetilde{\omega}(z)=z^{n+2} e^{2 i \theta}\left[\frac{(n+2) z^{n}+n z^{n-2}+2 e^{-i \theta}}{2 e^{i \theta} z^{n}+n z^{2}+(n+2)}\right]=z^{n+2} e^{2 i \theta} \frac{\beta(z)}{\beta^{*}(z)},
$$

where $\beta(z)=(n+2) z^{n}+n z^{n-2}+2 e^{-i \theta}$. To prove that $|\widetilde{\omega}(z)|<1$ in $E$, it suffices to show that all the zeros of $\beta$ lie inside or on $|z|=1$. The repeated application of Lemma 2.1 (as in the exceptional cases discussed above) allows 
us to conclude that this is in fact true. We skip the details for want of space. This completes the proof.

TABLE 1. Values of non-zero determinants in Case 3.

\begin{tabular}{|c|l|}
\hline Determinant & \multicolumn{1}{c|}{ Value } \\
\hline$\left|F_{1} E_{2} \cdots F_{n-1} E_{n} F_{n+1}\right|$ & $L_{n+1}\left[(2 n+1)^{2}\right]$ \\
\hline$\left|H_{1} E_{2} \cdots F_{n-1} E_{n} F_{n+1}\right|$ & $L_{n+1}[-a(2 n+1)(2 n-1)]$ \\
\hline$\left|G_{1} E_{2} \cdots F_{n-1} E_{n} G_{n+1}\right|$ & $L_{n+1}\left[\frac{1}{2}(2 n-1)(n+1)(2 a+a n-n)\right]$ \\
\hline$\left|G_{1} E_{2} \cdots G_{n-1} E_{n} F_{n+1}\right|$ & $L_{n+1}\left[\frac{1}{2}(2 n-1)(n-1)(n-2-a n)\right]$ \\
\hline
\end{tabular}

TABLE 2. Values of non-zero determinants in Case 4.

\begin{tabular}{|l|l|l|}
\hline \multirow{2}{*}{ Determinant } & \multicolumn{1}{|c|}{ Value } \\
\cline { 2 - 3 } & \multicolumn{1}{|c|}{ when $\mathbf{n} / \mathbf{2}$ is odd } & \multicolumn{1}{c|}{ when $\mathbf{n} / \mathbf{2}$ is even } \\
\hline$\left|F_{1} E_{2} \cdots F_{n-1} E_{n} F_{n+1}\right|$ & $L_{n+1}[2 n(2 n+2)]$ & $L_{n+1}[2 n(2 n+2)]$ \\
\hline$\left|F_{1} E_{2} \cdots F_{n-1} E_{n} G_{n+1}\right|$ & $L_{n+1}\left[e^{i \theta}(2 a+a n-n) n^{2}\right]$ & $L_{n+1}\left[-e^{i \theta}(2 a+a n-n) n^{2}\right]$ \\
\hline$\left|F_{1} E_{2} \cdots G_{n-1} E_{n} F_{n+1}\right|$ & $L_{n+1}\left[e^{i \theta}(n-a n-2) n(n+2)\right]$ & $L_{n+1}\left[-e^{i \theta}(n-a n-2) n(n+2)\right]$ \\
\hline$\left|G_{1} E_{2} \cdots F_{n-1} E_{n} F_{n+1}\right|$ & $L_{n+1}\left[-e^{-i \theta} 4 n\right]$ & $L_{n+1}\left[e^{-i \theta} 4 n\right]$ \\
\hline$\left|G_{1} E_{2} \cdots G_{n-1} E_{n} F_{n+1}\right|$ & $L_{n+1}[n(n-2)(n-a n-2)]$ & $L_{n+1}[n(n-2)(n-a n-2)]$ \\
\hline$\left|G_{1} E_{2} \cdots F_{n-1} E_{n} G_{n+1}\right|$ & $L_{n+1}\left[(2 a+a n-n) n^{2}\right]$ & $L_{n+1}\left[(2 a+a n-n) n^{2}\right]$ \\
\hline$\left|H_{1} E_{2} \cdots F_{n-1} E_{n} F_{n+1}\right|$ & $L_{n+1}\left[-a(2 n)^{2}\right]$ & $L_{n+1}\left[-a(2 n)^{2}\right]$ \\
\hline
\end{tabular}

TABLE 3. Values of non-zero determinants in Case 5.

\begin{tabular}{|c|l|}
\hline Determinant & \multicolumn{1}{|c|}{ Value } \\
\hline$\left|F_{1} F_{2} \cdots F_{n-1} F_{n} F_{n+1} F_{n+2}\right|$ & $L_{n+2}[(2 n+1)(2 n+3)]$ \\
\hline$\left|F_{1} F_{2} \cdots F_{n-1} F_{n} G_{n+1} G_{n+2}\right|$ & $L_{n+2}\left[\frac{e^{2 \imath \theta}}{4} n^{2}(n-2 a-a n)^{2}\right]$ \\
\hline$\left|F_{1} F_{2} \cdots F_{n-1} G_{n} G_{n+1} F_{n+2}\right|$ & $L_{n+2}\left[-\frac{e^{22 \theta}}{4} n(n+2)(n-2 a-a n)(n-2-a n)\right]$ \\
\hline$\left|F_{1} F_{2} \cdots G_{n-1} F_{n} F_{n+1} G_{n+2}\right|$ & $L_{n+2}\left[-\frac{e^{22 \theta}}{4} n(n+2)(n-2 a-a n)(n-2-a n)\right]$ \\
\hline$\left|F_{1} F_{2} \cdots G_{n-1} G_{n} F_{n+1} F_{n+2}\right|$ & $L_{n+2}\left[\frac{e^{2 \ell \theta}}{4}(n+2)^{2}(n-2-a n)^{2}\right]$ \\
\hline$\left|F_{1} G_{2} \cdots F_{n-1} F_{n} F_{n+1} G_{n+2}\right|$ & $L_{n+2}\left[-\frac{1}{2}(n-2 a-a n)(2 n-1)(n+3)\right]$ \\
\hline$\left|F_{1} G_{2} \cdots F_{n-1} G_{n} F_{n+1} F_{n+2}\right|$ & $L_{n+2}\left[\frac{1}{2}(n-2-a n)(2 n-1)(n+1)\right]$ \\
\hline$\left|F_{1} H_{2} \cdots F_{n-1} F_{n} F_{n+1} F_{n+2}\right|$ & $L_{n+2}[-a(2 n-1)(2 n+3)]$ \\
\hline$\left|G_{1} F_{2} \cdots F_{n-1} F_{n} G_{n+1} F_{n+2}\right|$ & $L_{n+2}\left[-\frac{1}{2}(n-2 a-a n)(2 n+1)(n+1)\right]$ \\
\hline$\left|G_{1} F_{2} \cdots G_{n-1} F_{n} F_{n+1} F_{n+2}\right|$ & $L_{n+2}\left[\frac{1}{2}(n-2-a n)(2 n+1)(n-1)\right]$ \\
\hline$\left|G_{1} G_{2} \cdots F_{n-1} F_{n} F_{n+1} F_{n+2}\right|$ & $L_{n+2}\left[4 e^{-2 n \theta}\right]$ \\
\hline$\left|G_{1} G_{2} \cdots F_{n-1} F_{n} G_{n+1} G_{n+2}\right|$ & $L_{n+2}\left[\frac{1}{4}(n-2 a-a n)^{2}\left(n^{2}-1\right)\right]$ \\
\hline$\left|G_{1} G_{2} \cdots F_{n-1} G_{n} G_{n+1} F_{n+2}\right|$ & $L_{n+2}\left[-\frac{1}{4}(n-2 a-a n)(n-2-a n)(n-1)^{2}\right]$ \\
\hline$\left|G_{1} G_{2} \cdots G_{n-1} F_{n} F_{n+1} G_{n+2}\right|$ & $L_{n+2}\left[-\frac{1}{4}(n-2 a-a n)(n-2-a n)(n-3)(n+1)\right]$ \\
\hline$\left|G_{1} G_{2} \cdots G_{n-1} G_{n} F_{n+1} F_{n+2}\right|$ & $L_{n+2}\left[\frac{1}{4}(n-2-a n)^{2}(n-3)(n-1)\right]$ \\
\hline$\left|G_{1} H_{2} \cdots F_{n-1} F_{n} G_{n+1} F_{n+2}\right|$ & $L_{n+2}\left[\frac{1}{2} a(n-2 a-a n)(n-1)(2 n+1)\right]$ \\
\hline$\left|G_{1} H_{2} \cdots G_{n-1} F_{n} F_{n+1} F_{n+2}\right|$ & $L_{n+2}\left[-\frac{1}{2} a(n-2-a n)(n-3)(2 n+1)\right]$ \\
\hline$\left|H_{1} F_{2} \cdots F_{n-1} F_{n} F_{n+1} F_{n+2}\right|$ & $L_{n+2}\left[-a(2 n+1)^{2}\right]$ \\
\hline$\left|H_{1} G_{2} \cdots F_{n-1} F_{n} F_{n+1} G_{n+2}\right|$ & $L_{n+2}\left[\frac{1}{2} a(n-2 a-a n)(n+1)(2 n-1)\right]$ \\
\hline$\left|H_{1} G_{2} \cdots F_{n-1} G_{n} F_{n+1} F_{n+2}\right|$ & $L_{n+2}\left[-\frac{1}{2} a(n-2-a n)(n-1)(2 n-1)\right]$ \\
\hline$\left|H_{1} H_{2} \cdots F_{n-1} F_{n} F_{n+1} F_{n+2}\right|$ & $L_{n+2}\left[a^{2}(2 n+1)(2 n-1)\right]$ \\
\hline
\end{tabular}

Acknowledgement. Authors are grateful to the referee for his valuable comments. The first author is also thankful to the Council of Scientific and Industrial Research, New Delhi, for financial support vide grant no.09/797/0006/2010 EMR-1. 
TABLe 4. Values of non-zero determinants in Case 6.

\begin{tabular}{|c|c|c|}
\hline \multirow[t]{2}{*}{ Determinant } & \multicolumn{2}{|c|}{ Value } \\
\hline & when $\mathbf{n} / \mathbf{2}$ is odd & when $\mathbf{n} / \mathbf{2}$ is even \\
\hline$\left|F_{1} F_{2} \cdots F_{n-1} F_{n} F_{n+1} F_{n+2}\right|$ & $L_{n+2}\left[(2 n+2)^{2}\right]$ & $L_{n+2}\left[(2 n+2)^{2}\right]$ \\
\hline$\left|F_{1} F_{2} \cdots F_{n-1} F_{n} F_{n+1} G_{n+2}\right|$ & $L_{n+2}\left[-\frac{e^{i v}}{2}(n-2 a-a n) n(2 n+2)\right]$ & $L_{n+2}\left[\frac{e^{\theta t}}{2}(n-2 a-a n) n(2 n+2)\right]$ \\
\hline$\left|F_{1} F_{2} \cdots F_{n-1} F_{n} G_{n+1} F_{n+2}\right|$ & $L_{n+2}\left[-\frac{e^{2 \theta}}{2}(n-2 a-a n) n(2 n+2)\right]$ & $L_{n+2}\left[\frac{e^{2 \theta}}{2}(n-2 a-a n) n(2 n+2)\right]$ \\
\hline$\left|F_{1} F_{2} \cdots F_{n-1} F_{n} G_{n+1} G_{n+2}\right|$ & $L_{n+2}\left[\frac{e^{2 i \ell}}{4}(n-2 a-a n)^{2} n^{2}\right]$ & $L_{n+2}\left[\frac{e^{2 i \theta}}{4}(n-2 a-a n)^{2} n^{2}\right]$ \\
\hline$\left|F_{1} F_{2} \cdots F_{n-1} G_{n} F_{n+1} F_{n+2}\right|$ & $L_{n+2}\left[\frac{e^{\frac{2 t}{2}}}{2}(n-2-a n)(2 n+2)(n+2)\right]$ & $L_{n+2}\left[-\frac{e^{i \theta}}{2}(n-2-a n)(2 n+2)(n+2)\right]$ \\
\hline$\left|F_{1} F_{2} \cdots F_{n-1} G_{n} G_{n+1} F_{n+2}\right|$ & $L_{n+2}\left[-\frac{e^{2 i \theta}}{4}(n-2 a-a n)(n-2-a n)(n+2) n\right]$ & $L_{n+2}\left[-\frac{e^{22 \theta}}{4}(n-2 a-a n)(n-2-a n)(n+2) n\right]$ \\
\hline$\left|F_{1} F_{2} \cdots G_{n-1} F_{n} F_{n+1} F_{n+2}\right|$ & $L_{n+2}\left[\frac{e^{\imath \theta}}{2}(n-2-a n)(2 n+2)(n+2)\right]$ & $L_{n+2}\left[-\frac{e^{2} \theta}{2}(n-2-a n)(2 n+2)(n+2)\right]$ \\
\hline$\left|F_{1} F_{2} \cdots G_{n-1} F_{n} F_{n+1} G_{n+2}\right|$ & $L_{n+2}\left[-\frac{e^{22 \theta}}{4}(n-2 a-a n)(n-2-a n)(n+2) n\right]$ & $L_{n+2}\left[-\frac{e^{2+\theta}}{4}(n-2 a-a n)(n-2-a n)(n+2) n\right]$ \\
\hline$\left|F_{1} F_{2} \cdots G_{n-1} G_{n} F_{n+1} F_{n+2}\right|$ & $L_{n+2}\left[\frac{e^{2 x t}}{4}(n-2-a n)^{2}(n+2)^{2}\right]$ & $L_{n+2}\left[\frac{e^{2, \theta}}{4}(n-2-a n)^{2}(n+2)^{2}\right]$ \\
\hline$\left|F_{1} G_{2} \cdots F_{n-1} F_{n} F_{n+1} F_{n+2}\right|$ & $L_{n+2}\left[-2 e^{-i \theta}(2 n+2)\right]$ & $L_{n+2}\left[2 e^{-i \theta}(2 n+2)\right]$ \\
\hline$\left|F_{1} G_{2} \cdots F_{n-1} F_{n} F_{n+1} G_{n+2}\right|$ & $L_{n+2}[-(n-2 a-a n) n(n+1)]$ & \begin{tabular}{|l}
$L_{n+2}[-(n-2 a-a n) n(n+1)]$ \\
\end{tabular} \\
\hline$\left|F_{1} G_{2} \cdots F_{n-1} F_{n} G_{n+1} F_{n+2}\right|$ & $L_{n+2}[(n-2 a-a n) n]$ & $L_{n+2}[(n-2 a-a n) n]$ \\
\hline$\left|F_{1} G_{2} \cdots F_{n-1} F_{n} G_{n+1} G_{n+2}\right|$ & $L_{n+2}\left[\frac{e^{i \theta}}{4}(n-2 a-a n)^{2} n^{2}\right]$ & $L_{n+2}\left[-\frac{e^{i \theta}}{4}(n-2 a-a n)^{2} n^{2}\right]$ \\
\hline$\left|F_{1} G_{2} \cdots F_{n-1} G_{n} F_{n+1} F_{n+2}\right|$ & $L_{n+2}[(n-2-a n)(n-2)(n+1)]$ & $L_{n+2}[(n-2-a n)(n-2)(n+1)]$ \\
\hline$\left|F_{1} G_{2} \cdots F_{n-1} G_{n} G_{n+1} F_{n+2}\right|$ & $L_{n+2}\left[-\frac{e^{2 \theta}}{4}(n-2 a-a n)(n-2-a n)(n-2) n\right]$ & $L_{n+2}\left[\frac{e^{2 \theta \theta}}{4}(n-2 a-a n)(n-2-a n)(n-2) n\right]$ \\
\hline$\left|F_{1} G_{2} \cdots G_{n-1} F_{n} F_{n+1} F_{n+2}\right|$ & $L_{n+2}[-(n-2-a n)(n+2)]$ & $L_{n+2}[-(n-2-a n)(n+2)]$ \\
\hline$\left|F_{1} G_{2} \cdots G_{n-1} F_{n} F_{n+1} G_{n+2}\right|$ & $L_{n+2}\left[-\frac{e^{2 \theta}}{4}(n-2 a-a n)(n-2-a n)(n+2) n\right]$ & $L_{n+2}\left[\frac{e^{2 \theta}}{4}(n-2 a-a n)(n-2-a n)(n+2) n\right]$ \\
\hline$\left|F_{1} G_{2} \cdots G_{n-1} G_{n} F_{n+1} F_{n+2}\right|$ & $L_{n+2}\left[\frac{e^{\imath t}}{4}(n-2-a n)^{2}(n-2)(n+2)\right]$ & $L_{n+2}\left[-\frac{e^{i \theta}}{4}(n-2-a n)^{2}(n-2)(n+2)\right]$ \\
\hline$\left|F_{1} H_{2} \cdots F_{n-1} F_{n} F_{n+1} F_{n+2}\right|$ & $\mid L_{n+2}[-4 a n(n+1)]$ & $\begin{array}{l}L_{n+2}[-4 a n(n+1)] \\
\end{array}$ \\
\hline$\left|F_{1} H_{2} \cdots F_{n-1} F_{n} G_{n+1} F_{n+2}\right|$ & {$\left[L_{n+2}\left[e^{i \theta} a(n-2 a-a n) n^{2}\right]\right.$} & $L_{n+2}\left[-e^{i \theta} a(n-2 a-a n) n^{2}\right]$ \\
\hline$\left|F_{1} H_{2} \cdots G_{n-1} F_{n} F_{n+1} F_{n+2}\right|$ & $L_{n+2}\left[-e^{i \theta} a(n-2-a n) n(n+2)\right]$ & $L_{n+2}\left[e^{i \theta} a(n-2-a n) n(n+2)\right]$ \\
\hline$\left|G_{1} F_{2} \cdots F_{n-1} F_{n} F_{n+1} F_{n+2}\right|$ & $L_{n+2}\left[-4 e^{-i \theta}(n+1)\right]$ & $L_{n+2}\left[4 e^{-i \theta}(n+1)\right]$ \\
\hline$\left|G_{1} F_{2} \cdots F_{n-1} F_{n} F_{n+1} G_{n+2}\right|$ & $L_{n+2}[(n-2 a-a n) n]$ & $L_{n+2}[(n-2 a-a n) n]$ \\
\hline$\left|G_{1} F_{2} \cdots F_{n-1} F_{n} G_{n+1} F_{n+2}\right|$ & $L_{n+2}[-(n-2 a-a n) n(n+1)]$ & $L_{n+2}[-(n-2 a-a n) n(n+1)]$ \\
\hline$\left|G_{1} F_{2} \cdots F_{n-1} F_{n} G_{n+1} G_{n+2}\right|$ & $L_{n+2}\left[\frac{e^{2 \theta}}{4}(n-2 a-a n)^{2} n^{2}\right]$ & $L_{n+2}\left[-\frac{e^{1 \theta}}{4}(n-2 a-a n)^{2} n^{2}\right]$ \\
\hline$\left|G_{1} F_{2} \cdots F_{n-1} G_{n} F_{n+1} F_{n+2}\right|$ & $L_{n+2}[-(n-2-a n)(n+2)]$ & $L_{n+2}[-(n-2-a n)(n+2)]$ \\
\hline$\left|G_{1} F_{2} \cdots F_{n-1} G_{n} G_{n+1} F_{n+2}\right|$ & $L_{n+2}\left[-\frac{e^{2 \theta}}{4}(n-2 a-a n)(n-2-a n) n(n+2)\right]$ & $L_{n+2}\left[\frac{e^{i \theta}}{4}(n-2 a-a n)(n-2-a n)\right.$ \\
\hline$\left|G_{1} F_{2} \cdots G_{n-1} F_{n} F_{n+1} F_{n+2}\right|$ & $L_{n+2}[(n-2-a n)(n-2)(n+1)]$ & $L_{n+2}[(n-2-a n)(n-2)(n+1)]$ \\
\hline$\left|G_{1} F_{2} \cdots G_{n-1} F_{n} F_{n+1} G_{n+2}\right|$ & $L_{n+2}\left[-\frac{e^{2 \theta}}{4}(n-2 a-a n)(n-2-a n) n(n-2)\right]$ & $L_{n+2}\left[\frac{e^{2 \theta}}{4}(n-2 a-a n)(n-2-a n) n(n-2)\right]$ \\
\hline$\left|G_{1} F_{2} \cdots G_{n-1} G_{n} F_{n+1} F_{n+2}\right|$ & $L_{n+2}\left[\frac{e^{2 \theta}}{4}(n-2-a n)^{2}(n+2)(n-2)\right]$ & $L_{n+2}\left[-\frac{e^{1 \theta}}{4}(n-2-a n)^{2}(n+2)(n-2)\right]$ \\
\hline$\left|G_{1} G_{2} \cdots F_{n-1} F_{n} F_{n+1} F_{n+2}\right|$ & $L_{n+2}\left[4 e^{-2 \imath \theta}\right]$ & $L_{n+2}\left[4 e^{-2 i \theta}\right]$ \\
\hline$\left|G_{1} G_{2} \cdots F_{n-1} F_{n} F_{n+1} G_{n+2}\right|$ & $\mid L_{n+2}\left[e^{-i \theta}(n-2 a-a n) n\right]$ & $L_{n+2}\left[-e^{-i \theta}(n-2 a-a n) n\right]$ \\
\hline$\left|G_{1} G_{2} \cdots F_{n-1} F_{n} G_{n+1} F_{n+2}\right|$ & $\mid L_{n+2}\left[e^{-i \theta}(n-2 a-a n) n\right]$ & $L_{n+2}\left[-e^{-\imath \theta}(n-2 a-a n) n\right]$ \\
\hline$\left|G_{1} G_{2} \cdots F_{n-1} F_{n} G_{n+1} G_{n+2}\right|$ & $L_{n+2}\left[\frac{1}{4}(n-2 a-a n)^{2} n^{2}\right.$ & $L_{n+2}\left[\frac{1}{4}(n-2 a-a n)^{2} n^{2}\right]$ \\
\hline$\left|G_{1} G_{2} \cdots F_{n-1} G_{n} F_{n+1} F_{n+2}\right|$ & $\mid L_{n+2}\left[-e^{-i \theta}(n-2-a n)(n-2)\right]$ & $\mid L_{n+2}\left[e^{-\imath \theta}(n-2-a n)(n-2)\right]$ \\
\hline$\left|G_{1} G_{2} \cdots F_{n-1} G_{n} G_{n+1} F_{n+2}\right|$ & $L_{n+2}\left[-\frac{1}{4}(n-2 a-a n)(n-2-a n) n(n-2)\right]$ & $L_{n+2}\left[-\frac{1}{4}(n-2 a-a n)(n-2-a n) n(n-2)\right]$ \\
\hline$\left|G_{1} G_{2} \cdots G_{n-1} F_{n} F_{n+1} F_{n+2}\right|$ & $L_{n+2}\left[-e^{-i \theta}(n-2-a n)(n-2)\right]$ & $L_{n+2}\left[e^{-i \theta}(n-2-a n)(n-2)\right]$ \\
\hline$\left|G_{1} G_{2} \cdots G_{n-1} F_{n} F_{n+1} G_{n+2}\right|$ & $L_{n+2}\left[-\frac{1}{4}(n-2 a-a n)(n-2-a n) n(n-2)\right]$ & $L_{n+2}\left[-\frac{1}{4}(n-2 a-a n)(n-2-a n) n(n-2)\right]$ \\
\hline$\left|G_{1} G_{2} \cdots G_{n-1} G_{n} F_{n+1} F_{n+2}\right|$ & $L_{n+2}\left[\frac{1}{4}(n-2-a n)^{2}(n-2)^{2}\right]$ & $L_{n+2}\left[\frac{1}{4}(n-2-a n)(n-2)^{2}\right]$ \\
\hline$\left|G_{1} H_{2} \cdots F_{n-1} F_{n} F_{n+1} F_{n+2}\right|$ & $L_{n+2}\left[4 e^{-i \theta} a n\right]$ & $L_{n+2}\left[-4 e^{-i \theta} a n\right]$ \\
\hline$\left|G_{1} H_{2} \cdots F_{n-1} F_{n} G_{n+1} F_{n+2}\right|$ & $L_{n+2}\left[a(n-2 a-a n) n^{2}\right]$ & $L_{n+2}\left[a(n-2 a-a n) n^{2}\right\rceil$ \\
\hline$\left|G_{1} H_{2} \cdots G_{n-1} F_{n} F_{n+1} F_{n+2}\right|$ & $\mid L_{n+2}[-a(n-2-a n) n(n-2)]$ & $L_{n+2}[-a(n-2-a n) n(n-2)]$ \\
\hline$\left|H_{1} F_{2} \cdots F_{n-1} F_{n} F_{n+1} F_{n+2}\right|$ & $L_{n+2}[-2 a n(2 n+2)]$ & $L_{n+2}[-2 a n(n+2)]$ \\
\hline$\left|H_{1} F_{2} \cdots F_{n-1} F_{n} F_{n+1} G_{n+2}\right|$ & $L_{n+2}\left[e^{i \theta} a n^{2}(n-2 a-a n)\right]$ & $L_{n+2}\left[-e^{\imath \theta} a n^{2}(n-2 a-a n)\right]$ \\
\hline$\left|H_{1} F_{2} \cdots F_{n-1} G_{n} F_{n+1} F_{n+2}\right|$ & $L_{n+2}\left[-e^{\imath \theta} a n(n+2)(n-2-a n)\right]$ & $L_{n+2}\left[e^{i \theta} a n(n+2)(n-2-a n)\right]$ \\
\hline$\left|H_{1} G_{2} \cdots F_{n-1} F_{n} F_{n+1} F_{n+2}\right|$ & $L_{n+2}\left[4 e^{-i \theta}\right.$ an $]$ & $L_{n+2}\left[-4 e^{-i \theta} a n\right]$ \\
\hline$\left|H_{1} G_{2} \cdots F_{n-1} F_{n} F_{n+1} G_{n+2}\right|$ & $L_{n+2}\left[a n^{2}(n-2 a-a n)\right]$ & $L_{n+2}\left[a n^{2}(n-2 a-a n)\right]$ \\
\hline$\left|H_{1} G_{2} \cdots F_{n-1} G_{n} F_{n+1} F_{n+2}\right|$ & $\begin{array}{l}L_{n+2}[-a n(n-2)(n-2-a n)] \\
\end{array}$ & $\begin{array}{l}L_{n+2}-a n(n-2)(n-2- \\
\end{array}$ \\
\hline$\left|H_{1} H_{2} \cdots F_{n-1} F_{n} F_{n+1} F_{n+2}\right|$ & $L_{n+2}\left[4 a^{2} n^{2}\right]$ & $L_{n+2}\left[4 a^{2} n^{2}\right]$ \\
\hline
\end{tabular}

\section{References}

[1] J. Clunie and T. Sheil-Small, Harmonic univalent functions, Ann. Acad. Sci. Fenn. Ser. A. I Math. 9 (1984), 3-25.

[2] M. Dorff, Harmonic univalent mappings onto asymmetric vertical strips, Computational methods and function theory 1997 (Nicosia), 171-175, Ser. Approx. Decompos., 11, World Sci. Publ., River Edge, NJ, 1999.

[3] Convolutions of planar harmonic convex mappings, Complex Var. Theory Appl. 45 (2001), no. 3, 263-271. 
[4] M. Dorff, M. Nowak, and M. Woloszkiewicz, Convolutions of harmonic convex mappings, Complex Var. Elliptic Equ. 57 (2012), no. 5, 489-503.

[5] W. Hengartner and G. Schober, Univalent harmonic functions, Trans. Amer. Math. Soc. 299 (1987), no. 1, 1-31.

[6] R. Kumar, M. Dorff, S. Gupta, and S. Singh, Convolution properties of some harmonic mappings in the right half-plane, see arXiv:1206.4364.

[7] R. Kumar, S. Gupta, S. Singh, and M. Dorff, An application of Cohn's rule to convolutions of univalent harmonic mappings, see arXiv:1306.5375.

[8] L. Li and S. Ponnusamy, Convolutions of slanted half-plane harmonic mappings, Analysis (Munich) 33 (2013), no. 2, 159-176.

[9] Sections of stable harmonic convex functions, Nonlinear Analysis 2014 (2014), 11 pages; http:/dx.doi.org/10.1016/j.na.2014.06.005.

[10] - Convolutions of harmonic mappings convex in one direction, Complex Anal. Oper. Th. 2014 (2014), 17 pages (available online: DOI 10.1007/s11785-014-0394-y).

[11] L. Li, S. Ponnusamy, and M. Vuorinen, The minimal surfaces over the slanted halfplanes, vertical strip and singlr slit, See http://arxiv.org/pdf/1204.2890.pdf

[12] Q. I. Rahman and G. Schmeisser, Analytic Theory of Polynomials, London Mathematical Society Monographs New Series, Vol. 26, Oxford University Press, Oxford, 2002.

RAJ KUMAR

Department of Mathematics

DAV UNIVERSITY

Jalandhar (Punjab), India

AND

Sant Longowal Institute of Engineering and Technology

LONGOWAL-148106 (PunJAB), INDiA

E-mail address: rajgarg2012@yahoo.co.in

Sushma Gupta

SAnt Longowal Institute of Engineering and Technology

LONGOWAL-148106 (Punjab), India

E-mail address: sushmagupta1@yahoo.com

Sukhjit Singh

SAnt Longowal Institute of Engineering and Technology

Longowal-148106 (Punjab), India

E-mail address: sukhjit_d@yahoo.com

Michael DorfF

Department of Mathematics

Brigham Young University

Provo, Utah, 84602, USA

E-mail address: mdorff@math.byu.edu 